TIFR/TH/07-11

\title{
Aspects of causal viscous hydrodynamics
}

\author{
R. S. Bhalerad* and Sourendu Gupta \\ Department of Theoretical Physics, \\ Tata Institute of Fundamental Research, \\ Homi Bhabha Road, Mumbai 400005, India.
}

\begin{abstract}
We investigate the phenomenology of freely expanding fluids, with different material properties, evolving through the Israel-Stewart (IS) causal viscous hydrodynamics, and compare our results with those obtained in the relativistic Eckart-Landau-Navier-Stokes (ELNS) acausal viscous hydrodynamics. Through the analysis of scaling invariants we give a definition of thermalization time which can be self-consistently determined in viscous hydrodynamics. Next we construct the solutions for one-dimensional boost-invariant flows. Expansion of viscous fluids is slower than that of one-dimensional ideal fluids, resulting in entropy production. At late times, these flows are reasonably well approximated by solutions obtained in ELNS hydrodynamics. Estimates of initial energy densities from observed final values are strongly dependent on the dynamics one chooses. For the same material, and the same final state, IS hydrodynamics gives the smallest initial energy density. We also study fluctuations about these one-dimensional boost-invariant backgrounds; they are damped in ELNS hydrodynamics but can become sound waves in IS hydrodynamics. The difference is obvious in power spectra due to clear signals of wave-interference in IS hydrodynamics, which is completely absent in ELNS dynamics.
\end{abstract}

PACS numbers: 25.75.-q, 24.10.Nz, 25.75.Ld, 12.38.Mh

\section{INTRODUCTION}

Hydrodynamics is an effective long-distance theory of fluids close to thermal and chemical equilibrium. The hydrodynamic modes are the fields which enter the theory, and can be identified from the symmetries of the microscopic theory. They include the energy-momentum tensor and densities of conserved quantities such as the baryon number and electric charge. The theory contains several unknown quantities, the transport coefficients and relaxation times, which one must obtain from microscopic computations in kinetic theory [1], or through measurements.

The relativistic version of the Navier-Stokes equation was first explored by Eckart 2] and subsequently by Landau [3], who developed what we call the ELNS formalism. The relativistic equations for ideal fluids are widely used in the contexts of heavy-ion collisions [4, 5, , 6, 7] and cosmology [8]. It has been suggested that the fluid produced in heavyion collisions at RHIC is very close to ideal. Such a claim must be substantiated by a study of viscous fluid dynamics. Until now there have been very few studies of non-ideal fluids in this context [9, 10, 11, 12, 13, 14, 15, 16, 17].

ELNS theory for non-ideal fluids is known to violate causality [18. The problem can be traced to the linear relation between fluxes and thermodynamic forces which is inherent in the Chapman-Enskog method of obtaining the ELNS equations from kinetic theory. It was realized by Israel and Stewart [19] that the problem with causality could be repaired by simply going beyond the linear relation between fluxes and forces. Their formulation of hydrodynamics is variously known as second-order theory, causal viscous hydrodynamics, or Israel-Stewart (IS) hydrodynamics. It contains an expanded set of material parameters when compared to ELNS theory.

There are other attempts to repair the loss of causality in Navier-Stokes theory. It was shown that the introduction of a phenomenologically motivated lag between the application of a thermodynamic force and the material response, through a memory kernel, could restore causality [20]. Such a phenomenological approach contains a smaller number of material parameters than IS hydrodynamics. In any case, little is known about some of the new parameters which appear in IS hydrodynamics. Even the relation between current correlation functions in a thermal quantum field theory and these quantities 21] has not been studied comprehensively.

In fact, once the problems of principle were resolved, further investigations of causal viscous hydrodynamics lagged because of an apparent paucity of applications. One expects that the main applications would be in situations where either the mean free path is comparable to the size of the region of interest or the relaxation time approaches the time scale of the phenomena of interest. It turns out that such applications are not hard to come by today. The former

\footnotetext{
*Electronic address: bhalerao@theory.tifr.res.in
}

†Electronic address: sgupta@tifr.res.in 
are possibly realized in heavy-ion collisions [12, 13, 14], and the diffusion of neutrinos through supernovae, the latter in astrophysical shock waves and freezeout in relativistic reactive fluids. Interest in such systems is on the increase.

Very little is presently known about the nature of fluid flows in IS hydrodynamics. The present paper is a step towards repairing this neglect, keeping future applications to heavy-ion physics in mind. In common with [11, 12, 13, 14] we investigate the equations for a fluid characterized completely by the energy-momentum tensor, i.e., neglecting net baryon number and electric charge, keeping only the shear part of the viscous stress tensor. We set up the equations in curvilinear coordinates appropriate to the approximate boost-invariant geometry of heavy-ion collisions, and reduce the tensor equations to coupled scalar equations. By examining the symmetries of the hydrodynamic equations we obtain laws of physical similarity.

Since an ideal fluid has zero viscosity and zero mean free path, the ideal hydrodynamic equations are unable to predict their own failure. All estimates of thermalization and freezeout in ideal hydrodynamics are imposed from outside. Since causal viscous hydrodynamics contains the relaxation time, $\tau_{\pi}$, one expects to improve upon this. We present a preliminary analysis.

Next we analyze boost-invariant solutions with three sets of constitutive relations for the fluid. In each case, we compare the ELNS and IS descriptions of boost-invariant flow and find that the former approximates the latter at late times. Our results for the case of the massless Boltzmann fluid are consistent with those given in [11, 12, 14]. We also examine the propagation of fluctuations around the boost-invariant solutions. Here the differences between ELNS and IS descriptions are remarkable: no propagating solutions exist in ELNS dynamics, whereas IS dynamics gives rise to damped sound waves.

The plan of the paper is as follows. The next section introduces the hydrodynamic equations and extracts scaling laws from them. This section also contains a discussion of the properties of materials that are needed in the remainder of the paper. Following this, we present the well-known Bjorken solution, and illustrate our method with the analysis of fluctuations around this solution for the ideal fluid. The next three sections examine a simple fluid, a Boltzmann fluid, and conformal fluids, respectively. In each case we examine the boost-invariant solution in IS hydrodynamics, its approach to ELNS hydrodynamics at late times, entropy production, and fluctuations around the boost-invariant solution. Through this analysis we build up a picture of general properties of the flow, as opposed to those which are specific to certain kinds of fluids. We summarize our understanding in the final section. The appendices contain details of the tensor analysis, the reduction of the tensor hydrodynamic equations to coupled scalar equations, and an analysis of transients.

\section{THE HYDRODYNAMIC EQUATIONS}

Throughout this paper we investigate the hydrodynamic equations in the limit of zero net quarks, i.e., vanishing baryon and charge density, since this is a good approximation to the actual situation realized in ultra-relativistic heavy-ion collisions. We also ignore the heat flux as in [11, 12, 13, 14]. References [22, 23] extend this to the case where the heat flux, thermal conductivity and baryon density are all included. It is strongly suspected that in the high-temperature phase of QCD, not very close to the crossover temperature $T_{c}$, the bulk viscosity is negligible. In view of this we investigate the equations where the bulk viscosity has been set to zero. With these simplifications the explicit form of the equations of Israel-Stewart (IS) hydrodynamics was written down in [11, 13, 14].

Having set the baryon and charge densities to zero, one is left with three independent hydrodynamic variables: a scalar, a vector and a tensor. The scalar is the energy density, $\epsilon$. It is related to the pressure, $p$, by the equation of state $-p=c_{s}^{2} \epsilon$, where $c_{s}$ is the speed of sound. Since all three quantities in the equation of state can be written as a function of the temperature, $T$, we sometimes trade $\epsilon$ for $T$. This variable specifies the part of the stress tensor from which external work can be extracted. Another of the hydrodynamic variables is the velocity 4 -vector $u^{\mu}$ (various choices of $u$ are discussed in [3] and [19]). Finally one has the dissipative part of the stress tensor. When bulk viscosity is neglected, this is a traceless symmetric tensor, $\pi^{\mu \nu}$. Since this part expresses shear, it must vanish on contraction with $u^{\mu}$.

Since we will discuss longitudinal flow, it is convenient to go from Galilean coordinates $(t, x, y, z)$ to the curvilinear coordinates $(\tau, \eta, r, \phi)$ where $\tau=\sqrt{t^{2}-z^{2}}, \eta=\tanh ^{-1}(z / t), r=\sqrt{x^{2}+y^{2}}$ and $\phi=\tan ^{-1}(y / x)$. The metric becomes $g_{\mu \nu}=\operatorname{diag}\left(1,-\tau^{2},-1,-r^{2}\right)$. The only non-vanishing Christoffel symbols are $\Gamma_{\eta \eta}^{\tau}=\tau, \Gamma_{\phi \phi}^{r}=-r, \Gamma_{\tau \eta}^{\eta}=\Gamma_{\eta \tau}^{\eta}=1 / \tau$ and $\Gamma_{r \phi}^{\phi}=\Gamma_{\phi r}^{\phi}=1 / r$ [24] . We will write covariant derivatives as $d_{\mu}$ and partial derivatives as $\partial_{\mu}$. The action of $d_{\mu}$ on a scalar field is the same as the action of $\partial_{\mu}$. In terms of the proper time $\mathcal{T}=\sqrt{\tau^{2}-r^{2}}$, one defines the components of the velocity 4 -vector $u^{\mu}=d x^{\mu} / d \mathcal{T}$. One can show that $u^{\mu} u_{\mu}=1$.

Longitudinal flow is an approximation applicable to ultra-relativistic heavy-ion collisions when the hydrodynamic variables at any point of spacetime depend on $\tau$ and $\eta$ but not on $r$ and $\phi$. Clearly such an approximation is valid far from the edges of the fluid volume, and at times $\tau \ll R / c_{s}$, where $R$ is a typical transverse size. We will parametrize 
the velocity vector by a quantity $y$ in the form-

$$
u^{\mu}=\left(\cosh y, \frac{1}{\tau} \sinh y, 0,0\right) .
$$

We note that the rapidity is $y+\eta$. Scaling flow corresponds to $y=0$ in our notation, as we discuss later. A fluid element with $y=0$, in our notation, moves along a world line of fixed $\eta$, corresponding to a constant velocity $v=\tanh \eta$. The divergence of $u$ is

$$
\Theta=d_{\mu} u^{\mu}=y_{\tau} \sinh y+\left(y_{\eta}+1\right) \frac{1}{\tau} \cosh y .
$$

This defines a macroscopic time scale for a hydrodynamic flow. Here, and later, we use the notation $f_{\tau}$ etc., to denote the derivative of a scalar $f$ with respect to the variable $\tau$ etc. We also define the material derivative $D=u^{\mu} d_{\mu}$, and through it the spacelike vector $D u^{\mu}$ and its norm $S^{2}=-D u^{\mu} D u_{\mu}$. A straightforward computation shows

$$
S=y_{\tau} \cosh y+\left(y_{\eta}+1\right) \frac{1}{\tau} \sinh y .
$$

Then it is easy to write down the unit space-like vector $v^{\mu}=\left(D u^{\mu}\right) / S$,

$$
v^{\mu}=\left(\sinh y, \frac{1}{\tau} \cosh y, 0,0\right) .
$$

One also defines another directional derivative operator $\widetilde{D}=v^{\mu} d_{\mu}$. In the local rest frame one finds that $D$ is the derivative with respect to time and $\widetilde{D}$ is the longitudinal spatial derivative.

Using the methods outlined in the appendices, one finds the hydrodynamic equations-

$$
\begin{aligned}
D \epsilon+B \Theta \epsilon & =\Theta \pi_{V}, \\
c_{s}^{2} \widetilde{D} \epsilon+B S \epsilon & =\widetilde{D} \pi_{V}+S \pi_{V}, \\
\tau_{\pi} D \pi_{V}+\pi_{V} & =\frac{4}{3} \eta_{V} \Theta,
\end{aligned}
$$

where $B=1+c_{s}^{2}, \eta_{V}$ is the coefficient of shear viscosity and $\tau_{\pi}$ is the relaxation time associated with the shear stress, $\pi_{V}$. Recall that such a relaxation time is necessary to construct causal hydrodynamics [18, 19]. For longitudinal flow we have reduced the tensor equations of hydrodynamics to three coupled scalar equations for the three scalar hydrodynamic quantities $\epsilon, y$ and $\pi_{V}$.

ELNS hydrodynamics is recovered when $\tau_{\pi}=0$, so that the last of eqs. (5) reduces to $\pi_{V}=4 \eta_{V} \Theta / 3$. One expects that for fluids which evolve inertially, i.e., in the absence of external forces acting during the evolution, the solutions of eqs. (5) should approach the solutions of ELNS hydrodynamics at times $\tau \gg \tau_{\pi}$. This conclusion may clearly change when a fluid is acted upon by external forces at all times. In such cases, of course, driving terms have to be added to the equations.

\section{A. Material properties at vanishing chemical potential}

For an ideal fluid, the equation of state can be cast into the form $p=c_{s}^{2} \epsilon$, where $p, \epsilon$ and $c_{s}$, could all be functions of the temperature, $T$. Straightforward dimensional analysis shows that $\epsilon=b T^{4}$, where $b$ is dimensionless. In general there are various intrinsic mass scales, $\mu_{i}$, in the fluid, and $b$ could have an implicit dependence on $T$ through the functional dependence, $b\left(T / \mu_{1}, T / \mu_{2}, \cdots\right)$. If $c_{s}^{2}=1 / 3$ at all temperatures, then the trace of the stress tensor vanishes identically. This implies a special symmetry called scale symmetry, or conformal symmetry [27]. One aspect of conformal symmetry is that uniform scaling of external scales such as $T$ by a constant leaves material properties unchanged. Clearly, then $b$ cannot depend on $T$, and must be constant.

For a non-ideal fluid, the stress tensor has an additional viscous part. When the trace of the full stress tensor vanishes, then the bulk viscosity vanishes identically. Conformal symmetry implies that the physics of such fluids can be expressed in terms of dimensionless combinations of material properties which are temperature independent. One such combination which has been used in the literature is $\eta_{V} / s$, where $\eta_{V}$ is the coefficient of shear viscosity and $s=(\epsilon+p) / T$ is the entropy density. In passing we note that for a conformal fluid, $s=\gamma \epsilon^{3 / 4}$, where $\gamma$ is a dimensionless constant.

Causal viscous hydrodynamics requires another material property of the fluid, the relaxation time for the shear part of the viscous stress tensor, $\tau_{\pi}$. Dimensionally, $\tau_{\pi}=a / T$, where $a$ is dimensionless, and becomes constant when 
the fluid has conformal symmetry. This dimensionless number is proportional to the quantity called liquidity [28] which, in non-relativistic fluids, measures the mean-free path (proportional to $\tau_{\pi}$ ) in units of the interparticle spacing (proportional to $1 / s^{1 / 3} \sim 1 / T$ ). In a gas, this number is very large, in liquids, small. It follows from the expressions for $s, \tau_{\pi}$ and the equation of state, that $s=\mathcal{K} \epsilon \tau_{\pi}$, where the dimensionless constant $\mathcal{K}=4 / 3 a$. This implies that the dimensionless material property $\epsilon \tau_{\pi} / \eta_{V}=s / \mathcal{K} \eta_{V}$. We call this combination $\chi$ and discuss it extensively in the next subsection.

In reality, the fluids that we are interested in are not conformal [29]. At temperatures below $T_{c}$ the fluid of hadron resonances has a plethora of mass scales, which breaks conformal symmetry, and manifests itself in deviations of $c_{s}^{2}$ from the value $1 / 3$. It is not a big stretch of the imagination to expect that bulk viscosity will be non-vanishing in this fluid. A fluid of quarks and gluons also breaks conformal symmetry through the conformal anomaly, which results in the running of the strong coupling and the appearance of the QCD scale $\Lambda_{Q C D}$, and explicitly through the quark masses. One question of interest is how important are these departures from conformal symmetry.

One might expect that at very large $T$, when the QCD coupling is close to zero, and all the quark masses are much less than $T$, one might have conformal symmetry to a good approximation, by virtue of the fluid being wellapproximated by a massless ideal gas. In fact, this is the limit in which the Boltzmann fluid approximation is seen to hold in weak coupling theory, with $\chi=3 / 2 c_{s}^{2}=9 / 2$. In this limit, one has, additionally, bulk viscosity much smaller than shear viscosity [30], $c_{s}^{2} \approx 1 / 3$, and the energy density close to an ideal gas value.

Lattice computations show, surprisingly, that approximate conformal symmetry is obtained also at substantially smaller $T / T_{c} \approx 2-3$, where the pressure deviates significantly from its ideal gas value [31]. Toy models of QCD with substantially enhanced ( $N=4$ super-) symmetries, which give up the running of the coupling and asymptotic freedom, have been used to model this observation. They are bound to fail in the vicinity of $T_{c}$ where the conformal measure [31] is large, and bulk viscosity cannot be neglected [32]. One prediction from these toy models, using the AdS/CFT conjecture, is that $\eta_{V} / s=1 / 4 \pi$, yielding $\chi=4 \pi / \mathcal{K}$. A recent computation in an appropriate $N=4 \mathrm{SYM}$ theory has found $a=(1-\ln 2) / 6 \pi[33]$, which then yields $\chi=(1-\ln 2) / 2 \approx 0.15$.

In this paper we shall examine three models of viscous fluids. The first, which we call a simple fluid, is one in which the material properties $c_{s}, \eta_{V}$ and $\tau_{\pi}$ are constant. Lattice results show that $c_{s}$ is almost constant over a range of $T$ 31]. However, preliminary lattice computations of transport coefficients are almost consistent with the power counting in $T$ over the same range [34]. As a result, the main motivation to study this model of a simple fluid is not its direct application to heavy-ion physics, but the fact that it allows explicit computation of the hydrodynamics, and contains qualitatively all the phenomena that we find with other models of viscous fluids, as we show in a later section.

The more restricted models of materials that we use have the property that $\chi$ is constant. A Boltzmann fluid has been examined in the literature [14], and is defined by the specific value $\chi=9 / 2$. We devote one section to detailed hydrodynamics of the Boltzmann fluid. In addition, in a subsequent section, we examine the whole class of conformal fluids with $\chi=3 \pi a$, for various $a$. Note that the hydrodynamics of the conformal fluid with $a=3 / 2 \pi$ (i.e., $\tau_{\pi}=3 / 2 \pi T$ ) is exactly equivalent to that for a Boltzmann fluid. As a result, it does not seem possible to use hydrodynamics alone to distinguish a conformal fluid from a Boltzmann fluid. We discuss this in greater detail later.

\section{B. Laws of physical similarity}

The equations of ideal hydrodynamics are obtained by setting $\pi_{V}=\eta_{V}=0$ in the eqs. (5). Then the equations for the remaining hydrodynamic variables, $\epsilon$ and $y$, are-

$$
D \epsilon+B \Theta \epsilon=0, \quad c_{s}^{2} \widetilde{D} \epsilon+B S \epsilon=0 .
$$

Consider the symmetries of these equations. The solutions are unchanged by the independent scalings $\epsilon \rightarrow \lambda \epsilon$ and $\tau \rightarrow \zeta \tau$. Introduce the variables $e=\ln \left(\epsilon / \epsilon_{0}\right)$ and $\theta=\ln \left(\tau / \tau_{0}\right)$, where the arbitrary scales $\epsilon_{0}$ and $\tau_{0}$ can be chosen to be the initial conditions. This removes the freedom of scaling, so that the solutions of the equations can be written in the form $e(\theta, \eta)$ and $y(\theta, \eta)$. Thus, the scale symmetries of the ideal hydrodynamic equations connect solutions with different initial conditions.

Non-ideal hydrodynamics breaks both these symmetries by the introduction of the time scale $\tau_{\pi}$ and the scale of energy density $\varpi=\eta_{V} / \tau_{\pi}$. In other words, the scaling $\epsilon \rightarrow \lambda \epsilon$ (simultaneously $\pi_{V} \rightarrow \lambda \pi_{V}$ ) and $\tau \rightarrow \zeta \tau$ are not symmetries unless one simultaneously scales $\tau_{\pi} \rightarrow \zeta \tau_{\pi}$ and $\eta_{V} \rightarrow \lambda \zeta \eta_{V}$. Thus, the scalings relate flows of fluids with different material properties. This is the relativistic analogue of scaling laws called "physical similarity" [3] that one finds in non-relativistic fluids. Such similarities are the basis of scaling invariants, also known as dimensionless variables, such as the Reynolds number, Re, which are used to relate flows of different fluids. 
The analysis here gives three scaling invariants-

$$
\chi=\frac{\epsilon}{\varpi}, \quad \varphi=\frac{\pi_{V}}{\varpi}, \quad \text { and } \quad \mathbf{S}=\frac{\pi_{V}}{\epsilon} .
$$

The dimensionless ratios lead to physical similarities between flows. We can relate these variables with quantities familiar from Navier-Stokes hydrodynamics by examining what they become in the appropriate limit.

As pointed out earlier, the ELNS limit of eqs. (5) is obtained when $\tau_{\pi}=0$. In that case, $\pi_{V}=4 \eta_{V} \Theta / 3$. The quantity $\Theta$ is the inverse of a characteristic scale for the flow, $\tau_{c}$. In the non-relativistic, i.e., the Navier-Stokes, limit, $\tau_{c}$ is a characteristic time scale. In this limit we can define a characteristic length scale for the flow by the relation $L_{c}=\tau_{c} v$, where $v$ is the flow velocity. Then, one finds

$$
\mathbf{S}=\frac{4 \eta_{V}}{3 \epsilon \tau_{c}}=\frac{4 c_{s}^{2}}{3}\left(\frac{v}{c_{s}}\right)^{2} \frac{\eta_{V}}{\epsilon L_{c} v}=\frac{4 c_{s}^{2}}{3} \frac{M^{2}}{\mathrm{Re}}
$$

where $M=v / c_{s}$ is the Mach number of the flow and Re $=\epsilon L_{c} v / \eta_{V}$ is the Reynolds number. The first of the expressions on the right comes from taking the ELNS limit, whereas the last expression involves taking, additionally, the non-relativistic limit. In Navier-Stokes hydrodynamics the ratio of inertial and viscous forces is Re [3]. In this case one may therefore suspect that $\mathbf{S} \propto 1 /$ Re. The exact relation above bears this out, with corrections needed to translate between the fully relativistic and non-relativistic formulas. Similarly, one finds that

$$
\varphi=\frac{4 \tau_{\pi}}{3 \tau_{c}}=\frac{4}{3 \xi} \frac{\lambda}{L_{c}} \frac{v}{c_{s}}=\frac{4}{3 \xi} M K
$$

where a mean-free path, $\lambda=\xi c_{s} \tau_{\pi}$, $\xi$ is some numerical constant, and $K=\lambda / L_{c}$ is the Knudsen number.

The third variable

$$
\chi=\frac{\varphi}{\mathbf{S}}=\frac{1}{c_{s}^{2} \xi} \frac{K \operatorname{Re}}{M}
$$

is interesting, since the combination $K \operatorname{Re} / M \simeq 1$ in Navier-Stokes theory. For Boltzmann and conformal fluids this combination is constant. The Navier-Stokes relation is obtained for these fluids when $\xi$ is chosen appropriately. For the Boltzmann fluid, this happens when $\xi \simeq 2 / 3$. Eqs. (8 9, 10) provide the connection between $\mathbf{S}, \varphi$ and $\chi$ and Re, $M$ and $K$ in the appropriate limit.

In ideal hydrodynamics thermalization and freezeout are notions which are imposed from the outside. In non-ideal hydrodynamics, however, some understanding of these phenomena could be possible [35]. In the Navier-Stokes theory, for example, $K \simeq M /$ Re must be smaller than unity in order for the solutions to describe valid flows. Since a solution of the Navier-Stokes equation allows us to compute both $M$ and Re, one can use the solution to compute $K$ and determine its own validity. A solution of the equations of IS hydrodynamics gives $\tau_{c}=1 / \Theta$. When $\tau_{c}$ is larger than $\tau_{\pi}$, i.e., when $\varphi<4 / 3$, the solution corresponds to a physical flow. In the case of scaling flow (discussed below), this gives an initial time, $\tau_{0}$, at which the solutions begin to describe physical fluid flows. Thus we have a self-consistent description of thermalization. For scaling flow, $\varphi$ decreases with time. Hence, after thermalization, IS hydrodynamics is always applicable; i.e., we lack a description of freezeout. That phenomenon requires us to examine radial flow. As argued before, radial flow becomes important at a time $\tau_{T}=R / c_{s}$, where $R$ is the transverse size. Thus, onedimensional IS hydrodynamics in the scaling approximation is expected to be valid in the range $\tau_{0} \leq \tau \leq \tau_{T}$. A description of freezeout at late times has to be sought in the full 3-dimensional hydrodynamics.

\section{Scaling solutions and fluctuations}

Solutions with $y=0$ are called scaling solutions or boost-invariant solutions. It was argued by Bjorken that asymptotic freedom implies that, at sufficiently high energies, hadron multiplicities must become invariant under longitudinal boosts. Assuming further that these multiplicities have their origin in the hydrodynamic distribution of the entropy density, he argued that the relevant flows in high energy heavy-ion collisions must be boost invariant [4]. While the phenomenological relevance of this argument may be questioned, boost invariance is simply analyzed. One uses $y=0$, as a consequence of which $S=0$ and $\Theta=1 / \tau$. Substituting these into eqs. (5), one finds that

$$
\tau D \epsilon=\pi_{V}-B \epsilon, \quad \widetilde{D} \pi_{V}=c_{s}^{2} \widetilde{D} \epsilon, \quad \tau D \pi_{V}=-\frac{\tau}{\tau_{\pi}} \pi_{V}+\frac{4}{3} \varpi,
$$

where $D=\partial_{\tau}$ and $\widetilde{D}=(1 / \tau) \partial_{\eta}$ (see eq. A4). Now, Fourier transforming in $\eta$ decouples the Fourier modes, labeled by $k$. The second equation implies that the identity $\pi_{V}(\tau, k)=c_{s}^{2} \epsilon(\tau, k)$ must hold for all $k \neq 0$. However the other 
two equations cannot be manipulated to give $D\left(\pi_{V} / \epsilon\right)=0$. Consequently, only the $k=0$ mode is allowed to be non-zero, i.e., the solutions to these three equations must have both $\epsilon$ and $\pi_{V}$ independent of $\eta$. This demonstrates the well-known equivalence of the conditions of boost invariance and $y=0$. Of course, the second of the three equations above becomes redundant and the problem can be treated with the remaining two equations.

In the next few sections we will investigate the scaling solutions for fluids with various different constitutive equations, and analyze their stability [5, 25]. Some numerical studies of the correlations of fluctuations were reported in [26]. We note that the IS hydrodynamic equations can be written in the form $\mathbf{x}_{\theta}=\mathbf{f}\left(\theta, \mathbf{x}, \mathbf{x}_{\eta}\right)$, where $\mathbf{x}$ is the vector of three hydrodynamic variables and $\theta$ is the "time" variable. Now, setting to zero the component corresponding to $y$ in $\mathbf{x}$ results in the boost-invariant equations, whose solutions we represent by $\mathbf{x}^{0}$. Represent the fluctuations around this solution by $\Delta \mathbf{x}^{1}$, where $\Delta \ll 1$ is a dimensionless book-keeping parameter. The equations for these fluctuations can be written to linear order in $\Delta$,

$$
\mathbf{x}_{\theta}^{1}=M_{0}\left(\theta, \mathbf{x}^{0}\right) \mathbf{x}^{1}+M_{1}\left(\theta, \mathbf{x}^{0}\right) \mathbf{x}_{\eta}^{1}
$$

where $M_{0}$ and $M_{1}$ are the Jacobian matrices of the derivatives of $\mathbf{f}$ with respect to $\mathbf{x}$ and $\mathbf{x}_{\eta}$ respectively. For the stability analysis one asks whether a given $\mathbf{x}^{1}$ increases or decreases with time. Fourier transforming in $\eta$ decouples the derivatives with respect to the variables and gives independent linear evolution equations for each mode $-\mathbf{x}_{\theta}^{1}(\theta, k)=$ $M \mathbf{x}^{1}(\theta, k)$, where $M=M_{0}+i k M_{1}$. The question of stability then reduces to examining $M$ and checking whether the solutions for $\mathbf{x}^{1}$ decrease faster than the scaling solution or not. We demonstrate the method with the ideal fluid in the next section.

\section{IDEAL FLUID: THE BJORKEN SOLUTION AND SOUND WAVES}
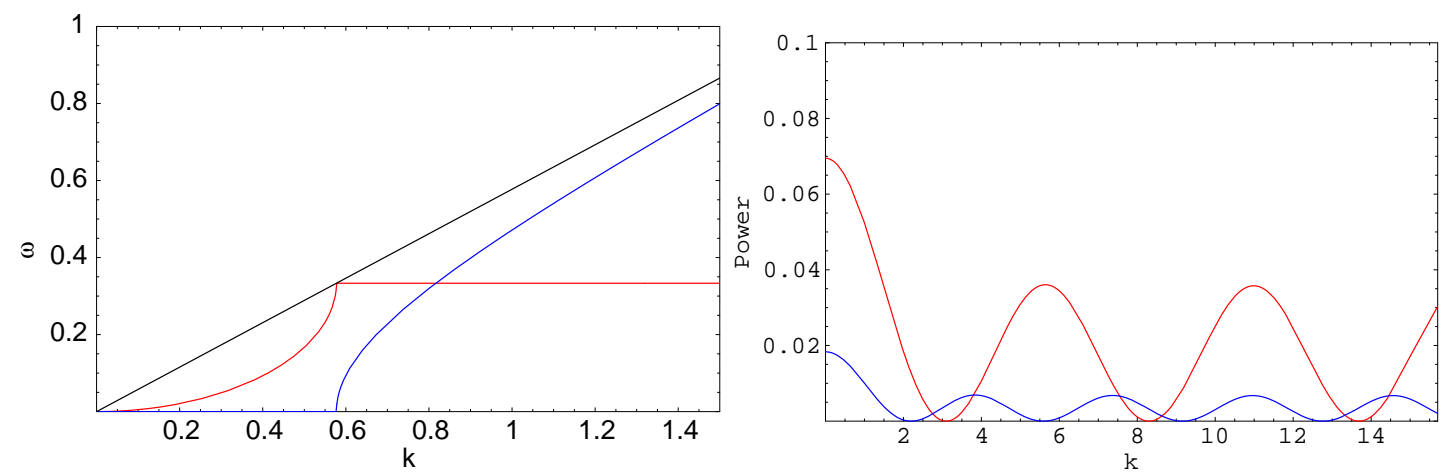

FIG. 1: (Color online) On the left is the dispersion relation for sound waves in the Bjorken solution for $c_{s}^{2}=1 / 3$. The real part of $\omega$ is shown in blue as a function of $k$ and the damping exponent, i.e., the imaginary part, is in red (the straight line in black is the line $\omega=c_{s} k$ ). There are no propagating waves for $k<1 / \sqrt{3}$. On the right is the power spectrum resulting from the evolution of $P_{\epsilon}(\theta=0 ; k)=1$ at $\theta=1$ (upper curve) and $3 / 2$ (lower curve).

The scaling solution in ideal hydrodynamics is obtained by setting $\eta_{V}=\pi_{V}=0$ in eqs. (11). Introducing the variables $e=\ln \left(\epsilon / \epsilon_{0}\right)$ and $\theta=\ln \left(\tau / \tau_{0}\right)$, the equations become-

$$
\frac{d e}{d \eta}=0, \quad \text { and } \quad \frac{d e}{d \theta}=-B
$$

This gives the Bjorken solution

$$
e(\theta, \eta)=-B \theta, \quad \text { i.e., } \quad \epsilon(\tau, \eta)=\epsilon_{0}\left(\frac{\tau_{0}}{\tau}\right)^{B} .
$$

The entropy density is defined as $s=(\epsilon+p) / T$. Entropy is conserved in an ideal fluid [3]. All this is well known.

The linearized equations for sound waves are extracted by introducing a dimensionless parameter $\Delta \ll 1$, which sets the scale of quantities involved in the propagation of sound relative to the boost-invariant background, as discussed earlier. Then one proceeds by setting $y(\theta, \eta)=\Delta y^{1}(\theta, \eta)$ and writing $e(\theta, \eta)=e^{0}(\theta)+\Delta e^{1}(\theta, \eta)$. Inserting these into the ideal fluid equations (6), one finds to order $\Delta^{0}$, the equations (13). At order $\Delta$ one finds-

$$
e_{\theta}^{1}=-B y_{\eta}^{1}, \quad \text { and } \quad y_{\theta}^{1}=-\left(1-\frac{1}{B}\right) e_{\eta}^{1}+(B-2) y^{1} .
$$


A Fourier transformation in $\eta$ reduces the partial differential equations above to uncoupled evolution equations for each Fourier mode. Since these linear first-order evolution equations are autonomous, i.e., they do not involve the variable $\theta$ anywhere explicitly, the solutions are in the form of waves $\exp [i(\omega \theta+k \eta)]$.

Before proceeding, a point about wave solutions may need comment. Plane waves in Galilean coordinates have the form $\exp [i(\omega t+\mathbf{k} \cdot \mathbf{x})]$, unlike the one above. However, the functional form of a wave solution is dependent on the geometry of the situation; for example, in spherical geometry a wave solution has the form $\exp [i(\omega t+k|\mathbf{x}|)] /|\mathbf{x}|$. Wave solutions in boost-invariant geometry have the specific form

$$
\mathrm{e}^{i(\omega \theta+k \eta)}=\left(\frac{\tau}{\tau_{0}}\right)^{i \omega} \mathrm{e}^{i k \eta}=\left(\frac{t+z}{\tau_{0}}\right)^{i(\omega+k) / 2}\left(\frac{t-z}{\tau_{0}}\right)^{i(\omega-k) / 2} .
$$

A real value for $\omega$ corresponds to an oscillatory solution. In the form on the right it is manifest that the real parts of $\omega$ and $k$ are not inverses of typical time and length scales for oscillation. If $\theta_{n}$ and $\theta_{n-1}$ are, respectively, the $n$-th and $n-1$ st times that the phase of the wave repeats at a given spacetime rapidity, then clearly $\theta_{n}-\theta_{n-1}=2 \pi / \omega$, so that $\tau_{n}=\tau_{n-1} \exp (2 \pi / \omega)$. In other words, the "period" of oscillation increases geometrically in the number of periods. The $n$-th time that the phase recurs after the initial time $\tau_{0}$ is given by $\tau_{n}=\tau_{0} \exp (2 \pi n / \omega)$. In the same way, at fixed $t$, the "wavelength" increases with $z$. Both these scalings are direct consequences of boost-invariant expansion- the longitudinal expansion red shifts sound waves. The analogy with the red shifting of light in an expanding universe is clear; both follow from the fact that the spatial components of the metric depend on the time. If $\omega$ is complex, then the real part gives oscillations exactly as described above. The imaginary part of $\omega$, i.e., $-\mathbf{R e} i \omega$, gives rise to power law behaviour in $\tau$. The scaling solution is stable when $\operatorname{Re} i \omega<0$, so that fluctuations are damped. Hence we will give the name damping exponent to $-\mathbf{R e} i \omega$.

Substituting the form of the wave solution in eq. (16) into eq. (15), one obtains the dispersion relations

$$
i \omega=-\frac{1}{2}\left(1-c_{s}^{2}\right) \pm \frac{1}{2} \sqrt{4 c_{s}^{2}\left(k_{0}^{2}-k^{2}\right)} \quad \text { where } \quad k_{0}=\frac{1-c_{s}^{2}}{2 c_{s}}
$$

For $k<k_{0}$ the modes are overdamped; the two damping exponents are equally spaced around $\left(1-c_{s}^{2}\right) / 2$. Only for $k>k_{0}$, are there propagating modes. These are damped due to the expansion of the scaling solution. The slowest decreasing part corresponds to the positive sign above. As a result, this is the part that is visible to experiments. This dispersion relation is shown in Figure 1. A general solution for $e^{1}$ can be written in the form

$$
e^{1}(\theta, k)=c_{+} \mathrm{e}^{i \omega_{+} \theta}+c_{-} \mathrm{e}^{i \omega_{-} \theta}
$$

where $c_{ \pm}$depend on the initial conditions, and $\omega_{ \pm}$are the solutions in eq. (17) with the corresponding signs. Similar solutions can be written for $y^{1}$. Since the real parts of $i \omega$ are non-positive, the fluctuations do not grow, and the scaling solution is stable [5, 25].

The physics of sound can be captured in the evolution of the power spectrum of fluctuations of the energy density,

$$
P_{\epsilon}(\tau ; k)=\left|\epsilon^{1}(\tau ; k)\right|^{2} \quad \text { where } \quad \epsilon^{1}(\tau ; k)=\int \frac{d \eta}{\sqrt{2 \pi}} \mathrm{e}^{-i k \eta} \epsilon^{1}(\tau, \eta)
$$

Since $\epsilon^{1}(\tau, \eta)=e^{1}(\tau, \eta) \epsilon^{0}(\tau)$, for the ideal fluid one may write asymptotically, when the component in $c_{-}$can be neglected,

$$
P_{\epsilon}(\tau ; k)=P_{\epsilon}\left(\tau_{0} ; k\right)\left(\frac{\tau_{0}}{\tau}\right)^{2 \omega_{d}}, \quad \text { where } \quad \omega_{d}(k)=B+\frac{1}{2}\left(1-c_{s}^{2}\right)-c_{s} \sqrt{k_{0}^{2}-k^{2}} \mathbf{H}\left(k_{0}-k\right),
$$

$k_{0}$ is given in eq. (17), and $\mathbf{H}$ denotes the unit step function. At not so late times, the interference between the frequencies $\omega_{+}$and $\omega_{-}$(when $k>k_{0}$ ) gives rise to beats. The shape of the power spectrum resulting from an initially flat power spectrum of fluctuations through exact solution of eqs. (15) with initial conditions $c_{+}=c_{-}$is shown in Figure 1. At short times it is dominated by beats. The expression in eq. (20) is asymptotic. As shown in Figure 1 , it is not recovered for $\theta=3 / 2$. It is clear that if the initial conditions contain fluctuations around the boost-invariant values, then after sufficient evolution, these fluctuations are damped. The longer the duration of hydrodynamic evolution, the more damped the fluctuations. A detailed analysis of the growth of transients at short times is given in Appendix C.

If the initial conditions, i.e., the power spectrum at time $\tau_{0}$, for an ideal fluid were known, then an event-by-event measurement of the power spectrum of the acoustic energy density would be able extract the value of $c_{s}$ and thereby give a measurement of the equation of state. Conversely, if the equation of state were known, then the same data could be used to extract, event by event, the initial conditions. 
The power spectrum studied here is closely related to the correlation function of fluctuations studied in [26]. In Fourier space the correlation function corresponds to studying the joint distribution of fluctuations at different $k$, whereas the power spectrum gives the variance in the fluctuations at a single $k$. Sonic peaks are visible in both the quantities. The analysis of the power spectrum in terms of interference, and the consequent clear relation with $c_{s}$, is a little harder to establish for the correlation function.

\section{A SIMPLE FLUID}

The simple non-ideal fluid model defined in Section II.A has constant values of all constitutive parameters- $c_{s}, \tau_{\pi}$ and $\eta_{V}$. Using the variables $\chi$ and $\varphi$ introduced in eq. (7) and $\vartheta=\tau / \tau_{\pi}$, we make the decomposition-

$$
\chi(\vartheta, \eta)=\chi^{0}(\vartheta)+\Delta \chi^{1}(\vartheta, \eta), \quad \varphi(\vartheta, \eta)=\varphi^{0}(\vartheta)+\Delta \varphi^{1}(\vartheta, \eta), \quad y(\vartheta, \eta)=\Delta y^{1}(\vartheta, \eta) .
$$

These expansions have to be substituted into the eqs. (5) and the material properties of the simple fluid used to extract equations for the boost-invariant solution $\chi^{0}$ and $\varphi^{0}$, and the fluctuations $\chi^{1}, y^{1}$ and $\varphi^{1}$. In the next two subsections we examine these two problems.

\section{A. The scaling solution}

The equations satisfied by the scaling solution are-

$$
\frac{d \chi^{0}}{d \vartheta}=-\frac{B}{\vartheta} \chi^{0}+\frac{\varphi^{0}}{\vartheta} \quad \text { and } \quad \frac{d \varphi^{0}}{d \vartheta}=-\varphi^{0}+\frac{4}{3 \vartheta} .
$$

One solves the second equation and then inserts the solution into the first. It is easy to check that

$$
\varphi^{0}(\vartheta)=\mathrm{e}^{-\vartheta}\left[b+\frac{4}{3} \operatorname{Ei}(\vartheta)\right], \quad b=-\frac{4}{3} \operatorname{Ei}\left(\vartheta_{0}\right)+\mathrm{e}^{\vartheta_{0}} \varphi^{0}\left(\vartheta_{0}\right)
$$

where $\operatorname{Ei}(x)$ denotes the exponential integral [36], and $\vartheta_{0}=\tau_{0} / \tau_{\pi}$. We will use $\vartheta_{0}=1$ in numerical work. The asymptotic expansion of the exponential integral,

$$
\operatorname{Ei}(x) \sim \frac{\mathrm{e}^{x}}{x}\left(1+\frac{1}{x}\right)
$$

can be used to write down the asymptotic expression-

$$
\varphi^{0}(\vartheta) \sim b \mathrm{e}^{-\vartheta}+\frac{4}{3 \vartheta}\left(1+\frac{1}{\vartheta}\right) .
$$

Note that the solution of the homogeneous equation decays much faster than the particular integral. Hence, the long-time behaviour of $\pi_{V}$ is nearly independent of the initial conditions on this quantity.
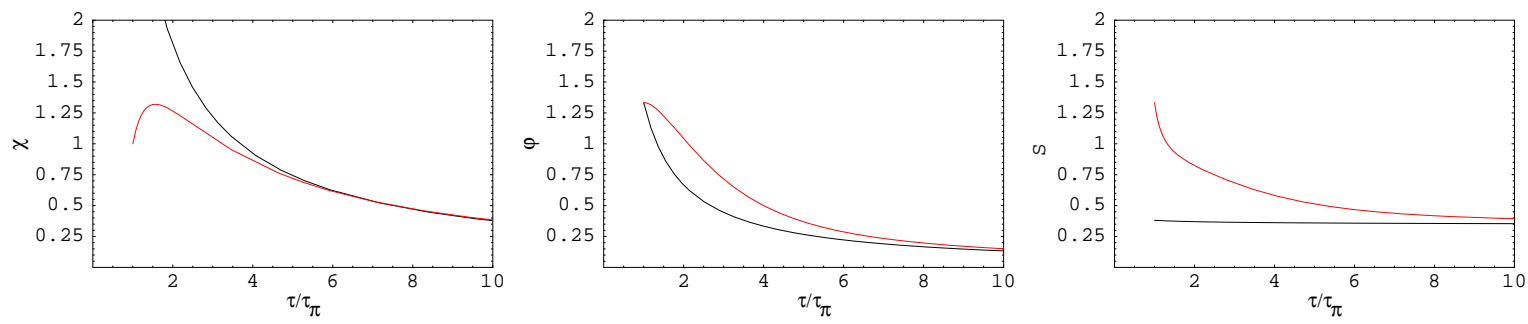

FIG. 2: (Color online) Comparison of ELNS hydrodynamics (lines in black) and IS hydrodynamics (in red). The evolution of normalized energy density, $\chi^{0}$, normalized shear stress, $\varphi^{0}$, and $\mathbf{S}=\varphi^{0} / \chi^{0}$, are shown as a function of the normalized time, $\tau / \tau_{\pi}$. The initial condition $\varphi^{0}(1)=4 / 3$ in both cases. 
Inserting the solution in eq. (23) into the first of eq. (22) gives a solution in terms of the Meijer G-functions [36, 37]. Instead, by inserting the asymptotic expansion in eq. (25) into the equation, we can find a simplified solution-

$$
\chi^{0}(\vartheta)=\frac{a}{\vartheta^{B}}-b \frac{\Gamma(B, \vartheta)}{\vartheta^{B}}+\frac{4}{3 c_{s}^{2} \vartheta}+\cdots
$$

where $a$ is determined by the initial condition $\chi^{0}\left(\vartheta_{0}\right)$ and the remaining terms decay as higher integer powers of $1 / \vartheta$. The incomplete Gamma function is defined to have the values $\Gamma(B, 0)=\Gamma(B)$ and $\Gamma(B, \infty)=0$. The solution of the homogeneous equation gives the Bjorken solution. However, this is not the slowest falling part; that is given by the $1 / \vartheta$ term, which arises from the inhomogeneous part of the equation, and has no dependence on initial conditions. In the very late time limit, one finds $\varphi^{0} \simeq 4 / 3 \vartheta$ and $\chi^{0} \simeq 4 / 3 c_{s}^{2} \vartheta$, so that the Bjorken solution is never recovered. The expansion is slowed down with respect to the ideal fluid because of dissipation - in expanding against the vacuum, an ideal fluid pumps all its energy into the expansion, whereas a non-ideal fluid dissipates energy, thus slowing the expansion. Similar results were also obtained in [9] for the case of ELNS hydrodynamics.

\section{Comparison of ELNS and IS hydrodynamics}

The differences between ELNS and IS hydrodynamics are illuminating. The ELNS limit of IS hydrodynamics is obtained formally by setting $\tau_{\pi}=0$, or, equivalently, by dropping the term $D \pi_{V}$ in eqs. (5). Now, a comparison of the two theories makes sense only after appropriate normalization of both time and energy. So in ELNS hydrodynamics one must introduce artificially a unit of time which is numerically equal to the value of $\tau_{\pi}$ used in IS hydrodynamics.

Then, rewriting the ELNS equations, one obtains scaled quantities $\chi^{0}$ and $\varphi^{0}$ which are directly comparable to the same quantities in IS. The equations of ELNS are then obtained from eqs. (22) by dropping the term $d \varphi^{0} / d \vartheta$, so that $\varphi^{0}=4 / 3 \vartheta$. Inserting this into the equation for $\chi^{0}$, one has

$$
\frac{d \chi^{0}}{d \vartheta}=-\frac{B \chi^{0}}{\vartheta}+\frac{4}{3 \vartheta^{2}}, \quad \text { so } \quad \chi^{0}(\vartheta)=\frac{4}{3(B-1) \vartheta}+\frac{\chi^{0}(1)-4 / 3(B-1)}{\vartheta^{B}} .
$$

A comparison of the resulting solutions is shown in Figure 2. The initial conditions for $\varphi^{0}$ are chosen to be the same; in IS hydrodynamics it corresponds to the smallest time at which the equations are valid, i.e., $\tau_{0}=\tau_{\pi}$. As in [11, 14], we find clear differences between ELNS and IS evolution. In earlier works the difference in the evolution of the energy density was investigated for equal initial conditions. We have chosen the initial conditions for $\chi^{0}$ to be different, but tuned so that the ELNS and IS solutions approach each other at large times. Since initial conditions are not detectable in heavy-ion collisions, it is of interest to see that large-time behaviour cannot, by itself, distinguish between ELNS and IS hydrodynamics. Moreover, the same final energy density can lead to different estimates of the initial energy density in the two kinds of dynamics.

\section{Entropy production}

Assuming that $s=\gamma \epsilon^{3 / 4}$, where $\gamma$ is a dimensionless quantity which is almost temperature independent, $\sigma=\left(\chi^{0}\right)^{3 / 4}$ is a dimensionless quantity proportional to the entropy density. The first of eqs. (22) can be easily manipulated into the form-

$$
\frac{d \sigma}{d \vartheta}=-\frac{\sigma}{\vartheta}+\frac{3 \varphi^{0}}{4 \vartheta \sigma^{1 / 3}}
$$

Using the solutions for $\varphi^{0}$ and $\chi^{0}$ in eqs. (25, 26), one finds that at late times the right hand side is negative. This is in accord with the exact solution for $\chi^{0}$ shown in Figure 2. Depending on the initial conditions, $\sigma$ may increase initially. However, at sufficiently large time it must decrease. If there is initial growth in $\sigma$, then the turnover comes when the right hand side of the above equation passes through zero, i.e., at the value of $\vartheta$ when $\chi^{0}=3 \varphi^{0} / 4$.

Note, however, that the element of 3 -volume contains a scale factor $\tau$ from the metric. This implies that the total entropy scales as $\Sigma=\sigma \vartheta$. It is a straightforward exercise to rewrite eq. (28) to obtain

$$
\frac{d \Sigma}{d \vartheta}=\frac{3 \varphi^{0}}{4}\left(\frac{\vartheta}{\Sigma}\right)^{1 / 3}
$$

The right hand side is manifestly positive definite, indicating that the total entropy increases with time. Using the asymptotic expansion of $\varphi^{0}$ in eq. (25), we find that $\Sigma$ grows asymptotically as $\vartheta^{1 / 4}$. One has the same power law growth of $\Sigma$ in ELNS dynamics. 


\section{B. Sound waves}

Using the decomposition of eq. (21), we examine small fluctuations around the scaling solution. At large $\vartheta$ we may use the asymptotic solutions $\chi^{0}=4 / 3 c_{s}^{2} \vartheta$ and $\varphi^{0}=4 / 3 \vartheta$ in the fluctuation equations. We also transform to the variable $\theta=\ln \vartheta$ and use $y^{1}=g \exp \theta$. After Fourier transforming in $\eta$, the equations for fluctuations take the form-

$$
\begin{aligned}
& \partial_{\theta}\left(\begin{array}{c}
\chi^{1} \\
g \\
\varphi^{1}
\end{array}\right)=M\left(\begin{array}{c}
\chi^{1} \\
g \\
\varphi^{1}
\end{array}\right), \quad \text { where } M=i k M_{1}+M_{0}, \\
& M_{1}=\left(\begin{array}{ccc}
0 & -\frac{4}{3 c_{s}^{2}} & 0 \\
-\frac{3 c_{s}^{4}}{4} & 0 & \frac{3 c_{s}^{2}}{4} \\
0 & \frac{4}{3} \mathrm{e}^{\theta} & 0
\end{array}\right), \quad M_{0}=\left(\begin{array}{ccc}
-B & 0 & 1 \\
0 & -2 & 0 \\
0 & 0 & -\mathrm{e}^{\theta}
\end{array}\right) .
\end{aligned}
$$

In contrast to the equations for fluctuations in an ideal fluid, i.e., eqs. (15), these equations are not autonomous. As a result, they cannot be solved by Fourier expansion in $\theta$. A numerical solution is always possible, and we can examine the limits of large and small $k$ analytically. At every $\tau$ there is an upper cutoff on $k$ imposed by the requirement of the applicability of hydrodynamics. This cutoff increases with $\tau$.
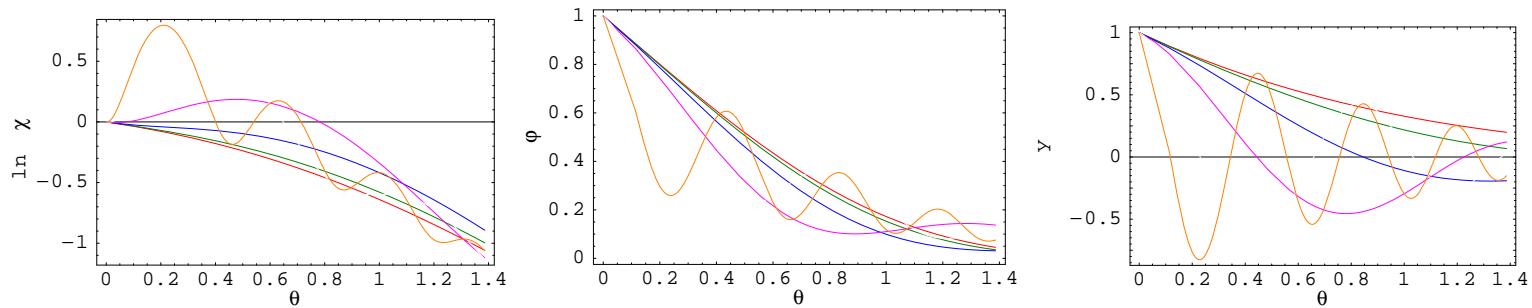

FIG. 3: (Color online) Solutions of the linearized equations for fluctuations around the scaling solution in a simple fluid. The normalized energy, $\chi^{1}$, shear, $\varphi^{1}$, and $y^{1}$ are shown for $k=1 / 2$ (red), 1 (green), 2 (blue), 4 (purple) and 8 (orange). The first three values of $k$ are overdamped, but the last two show oscillatory behaviour. The frequency of oscillation increases with $k$.
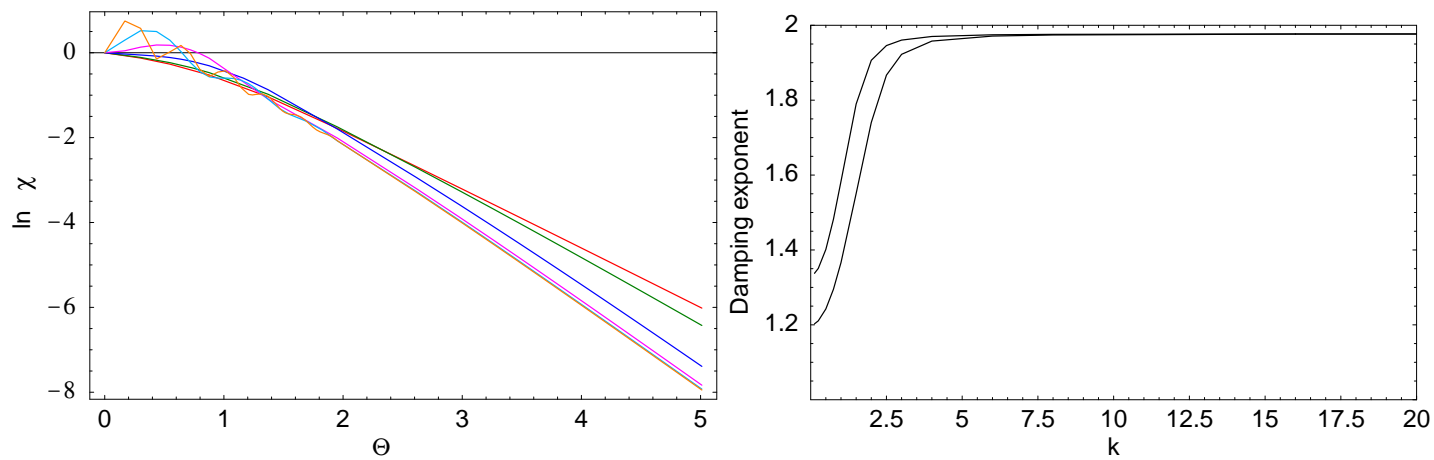

FIG. 4: (Color online) The panel on the left shows $\ln \chi^{1}(k, \tau)$ as a function of $\theta=\ln \left(\tau / \tau_{\pi}\right)$ for the simple fluid with $c_{s}^{2}=1 / 3$ for $k=1 / 2$ (red), 1 (green), 2 (blue), 4 (purple), 8 (light blue) and 16 (orange). The panel on the right shows the damping exponent, i.e., the imaginary part of $\omega$, for $c_{s}^{2}=1 / 5$ and $1 / 3$, plotted as a function of $k$.

In the limit $k \rightarrow 0$, one may set $M=M_{0}$. Then, since $M_{0}$ is diagonal, one can read off the solutions easily. $\chi^{1}$ and $g$ (and hence $y^{1}$ ) are overdamped, whereas $\varphi^{1}$ decays exponentially in $\tau$. The exact solutions are-

$$
\begin{aligned}
\chi^{1}(k \rightarrow 0, \tau) & =\chi^{1}\left(k \rightarrow 0, \tau_{0}\right)\left(\frac{\tau_{0}}{\tau}\right)^{B}-\varphi^{1}\left(k \rightarrow 0, \tau_{0}\right) \mathrm{e}^{\tau_{0} / \tau_{\pi}}\left(\frac{\tau_{\pi}}{\tau}\right)^{B}\left[\Gamma\left(B, \frac{\tau}{\tau_{\pi}}\right)-\Gamma\left(B, \frac{\tau_{0}}{\tau_{\pi}}\right)\right], \\
y^{1}(k \rightarrow 0, \tau) & =y^{1}\left(k \rightarrow 0, \tau_{0}\right)\left(\frac{\tau_{0}}{\tau}\right) \\
\varphi^{1}(k \rightarrow 0, \tau) & =\varphi^{1}\left(k \rightarrow 0, \tau_{0}\right) \exp \left(\frac{\tau_{0}-\tau}{\tau_{\pi}}\right) .
\end{aligned}
$$


In the regime $k \gg 1$, a first approximation would be to neglect $M_{0}$. As a result, one expects $\omega \propto k$, where $i \omega$ is an eigenvalue of $M$. Since $\omega$ is very large, $\exp \theta$ changes little over many oscillations. Consequently one could treat this factor as constant whenever it appears inside $M$. Within this approximation the equations above can be treated as autonomous and therefore generically describe oscillations.

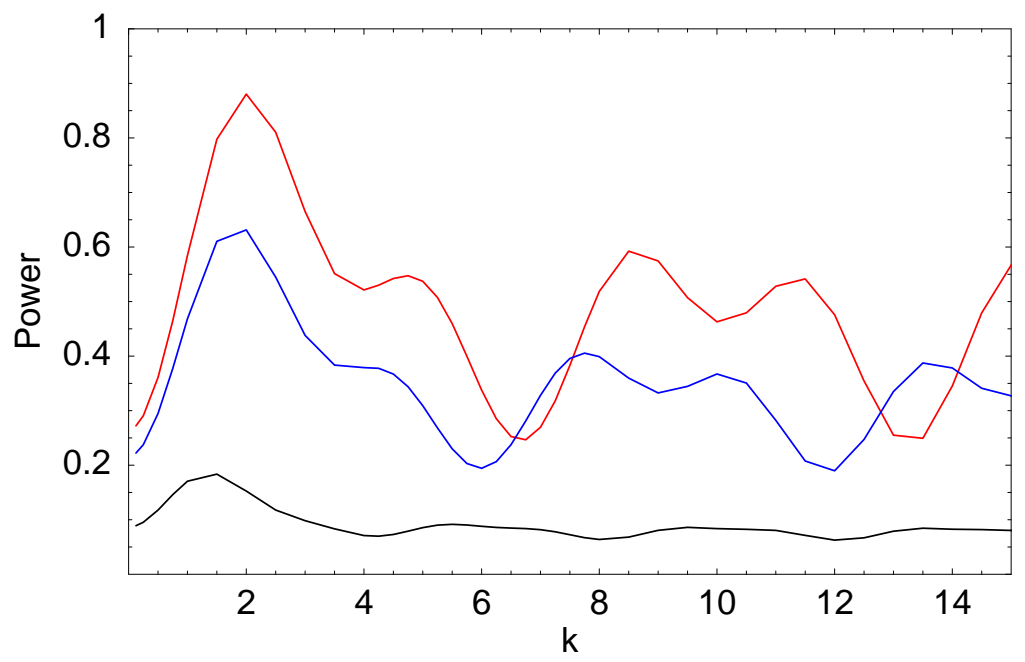

FIG. 5: (Color online) The power spectrum obtained from an initially flat power spectrum of fluctuations at $\theta=1$ (red), 1.1 (blue) and 1.5 (black). Here $c_{s}^{2}=1 / 3$ and $\tau_{0}$ has been set equal to $\tau_{\pi}$. Initial conditions are $\chi^{1}(0)=y^{1}(0)=\varphi^{1}(0)=1$.

Since the eigenvalues of $M_{1}$ are real, within the approximation where one neglects $M_{0}$, the solutions are not damped. To go beyond this and obtain the damping exponent, one sets up a perturbation theory in powers of $1 / k$ by writing $i \omega=i k \lambda_{1}+\lambda_{0}+\mathcal{O}(1 / k)$. The simplest way to proceed is to substitute this form into the characteristic equation for $M$. One finds then that this equation has a leading term of order $k^{3}$ (which solves the eigenvalue problem for $M_{1}$ ) and a first perturbation term of order $k^{2}$. The latter shows growing solutions in the unphysical region exp $\theta \ll 1$, but damping with $i \omega=-2+\mathcal{O}(1 / k)$ when $\exp \theta \gg 1$. Note that this damping exponent is independent of $c_{s}^{2}$.

The numerical solutions to eqs. (30) are shown for a range of $k$ in Figure 3 . There is clear evidence of overdamped solutions for $k \leq 2$ and damped oscillatory solutions for $k>2$. In Figure 4 we show that $\chi^{1}$, which is proportional to the energy density of fluctuations, is damped fairly rapidly. However, for $\theta \leq 1$ there are clear signs of transients; a detailed discussion of which is given in Appendix C. Numerically we see that $\chi^{1}$ is damped as a power of $\tau$ at large $\tau$, making it easy to extract the damping exponent numerically. As shown in the figure, and in agreement with our analysis above, at small $k$ this goes to $1+c_{s}^{2}$ and climbs to the neighbourhood of 2 at large $k$.

The power spectrum of fluctuations starting from an initially flat spectrum, $P_{\epsilon}(0 ; k)=1$, is easily amenable to computation, and is shown in Figure 5. The transient growth phenomenon gives rise to several peaks for $\theta \leq 1$. By $\theta \simeq 1.5$ the effect of the damping exponent is clearly visible. A complete analysis is given in Appendix $\mathrm{C}$.

\section{Sound in ELNS hydrodynamics}

Sound propagation in ELNS hydrodynamics is quite different. The equations for sound can be obtained from eqs. (30), by simply dropping the term $d \varphi^{1} / d \theta$, i.e., by setting $\varphi^{1}=4 i k g / 3$, and treating $\tau_{\pi}$ as an arbitrary scale of time, set equal to the value it would have in IS hydrodynamics. When this relation is inserted into the equation for $g_{\theta}$, a term in $g_{\eta \eta}$ is generated. This is the diffusive term we expect in ELNS hydrodynamics, and arises directly from the neglect of the relaxation time in shear pressure.

As before, we transform to variables $\theta=\ln \vartheta$ and write $y^{1}=g \exp \theta$. The equations for sound in ELNS hydrodynamics are

$$
\partial_{\theta}\left(\begin{array}{c}
\chi^{1} \\
g
\end{array}\right)=M\left(\begin{array}{c}
\chi^{1} \\
g
\end{array}\right), \quad \text { where } \quad M=-\left(\begin{array}{cc}
1+c_{s}^{2} & \frac{4\left(1-c_{s}^{2}\right) i k}{3 c_{s}^{2}} \\
\frac{3}{4} i c_{s}^{4} k & 2+c_{s}^{2} k^{2}
\end{array}\right) .
$$

In the limit of small $k$, i.e., $k \ll 1$, it is clear that $\chi^{1} \propto \tau^{-B}$ and $y^{1} \propto 1 / \tau$. This is similar to the results in eq. (31). In this limit the solutions of ELNS and IS hydrodynamics are not qualitatively different. This is expected since the characteristic time scale of the wave is much larger than $\tau_{\pi}$. 


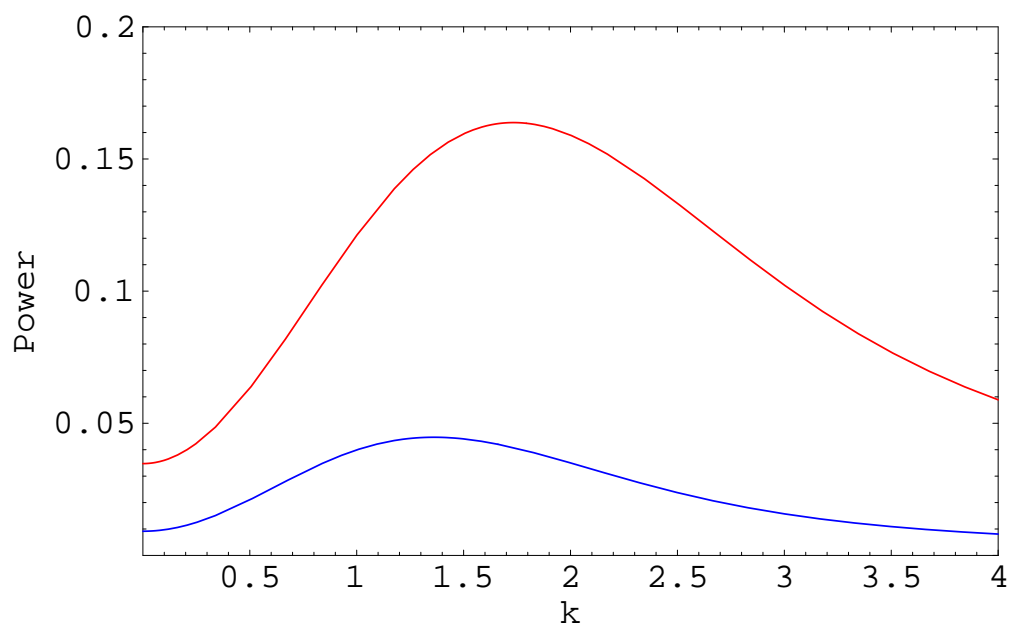

FIG. 6: (Color online) The power spectrum obtained in ELNS hydrodynamics for a simple fluid from an initially flat power spectrum of fluctuations at $\theta=2$ (red) and 2.5 (blue). Here $c_{s}^{2}=1 / 3$ and $\tau_{0}$ has been set equal to $\tau_{\pi}$.

In this case, an exact solution is possible for all $k$, since the equations are autonomous. The eigenvalues of $M$ are

$$
i \omega=\left\{\begin{array}{l}
-\left(B+c_{s}^{2} k^{2}\right) \\
-2,
\end{array}\right.
$$

where the combinations $4\left(1-c_{s}^{2}\right) \chi^{1} / 3 c_{s}^{4}-i k g$ and $4 i k \chi^{1} / 3 c_{s}^{2}+g$ decay respectively with these damping exponents. Not only is $y^{1}$ diffusively damped at large $k$, but there are no propagating modes at all. This behaviour is characteristic of parabolic equations.

In the absence of propagating modes there are no beats. Transient growth can occur, but there is only a single peak in the power spectrum of the fluctuations of energy. The transient analysis is given in Appendix C. The power spectrum resulting from an exact numerical solution, starting from $P_{\epsilon}(0 ; k)=1$, is shown in Figure 6. Comparing this with Figure 5, we see that there is a clear difference between diffusive damping of fluctuations in ELNS hydrodynamics and sound in IS dynamics.

\section{A BOLTZMANN FLUID}

A Boltzmann fluid is defined by the constitutive relation $\chi=\epsilon \tau_{\pi} / \eta_{V}=3 / 2 c_{s}^{2}=9 / 2$, where, as discussed in Section II.A, the three quantities $\epsilon, \eta_{V}$ and $\tau_{\pi}$, all depend on the temperature. We rewrite the hydrodynamic equations in terms of the variables $\mathbf{S}, y, u=T \tau /\left(T_{0} \tau_{0}\right)$, and $\theta=\ln \left(\tau / \tau_{0}\right)$, where the initial conditions are applied at the time $\tau_{0}$, i.e., at $\theta=0$. We analyze the scaling solution and its stability by the usual technique of writing $u(\tau, \eta)=u^{0}(\tau)+\Delta u^{1}(\tau, \eta)$, $\mathbf{S}(\tau, \eta)=\mathbf{S}^{0}(\tau)+\Delta \mathbf{S}^{1}(\tau, \eta)$ and $y(\tau, \eta)=\Delta y^{1}(\tau, \eta)$. Substituting these into eqs. (5), using the material properties, and separating out the equations to orders $\Delta^{0}$ and $\Delta$, we obtain the equations which lead to the scaling solution from the former, and the equations for fluctuations from the latter.

\section{A. The scaling solution}

The equations for the scaling flow become

$$
\partial_{\theta}\left(\begin{array}{c}
u \\
\mathbf{S}
\end{array}\right)=\mathbf{V}, \quad \text { where } \quad \mathbf{V}=\left(\begin{array}{c}
u\left[\mathbf{S}+3-c_{s}^{2}\right] / 4 \\
\mathcal{A}-\mathbf{S}^{2}+\mathbf{S}\left[\left(1+c_{s}^{2}\right)-\mathcal{B} u\right]
\end{array}\right)
$$

$\mathcal{A}=4 / 3 \chi, \mathcal{B}=\tau_{0} / \tau_{\pi}(0)$ and $c_{s}^{2}$ are non-negative. In this subsection we lighten the notation by writing $u$ for $u^{0}$ and $\mathbf{S}$ for $\mathbf{S}^{0}$. Three numbers are needed to fix the initial condition in the original formulation of the problem (eq. 5 ), i.e., the initial time $\tau_{0}$ and the values of $\epsilon\left(\tau_{0}\right)$ and $\pi_{V}\left(\tau_{0}\right)$. The initial condition on $u$ is, by definition, $u(\theta=0)=1$. The two free parameters in the initial conditions are transformed into the value of $\mathbf{S}(0)$ and the value of the parameter 
$\mathcal{B}$ which appears in the equation. Note that $\mathcal{B}<1$ is disfavoured. A dimensional quantity is needed to complete the specification of the initial conditions, and we can choose this to be $T_{0}=a \mathcal{B} / \tau_{0}$. Next, choosing $c_{s}^{2}=1 / 3$ (and hence $\chi=9 / 2$ ), one has $\mathcal{A}=8 / 27$. Since the equations are autonomous, one can analyze them using the phase plane method [38].

1. Phase plane structure
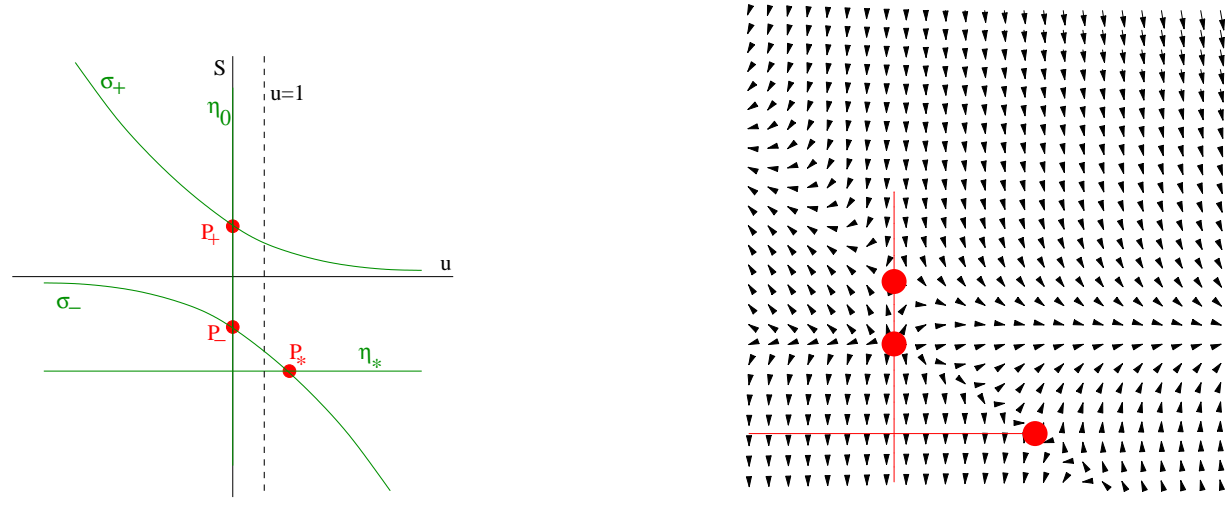

FIG. 7: (Color online) The panel on the left shows structural elements of the phase plane: the nullclines, i.e., the lines along which one of the derivatives vanishes (that of $u$ vanishes on $\eta_{0}$ and $\eta_{*}$, that of $\mathbf{S}$ vanishes on $\sigma_{ \pm}$), and the fixed points, at which both derivatives vanish. The panel on the right shows the vector field of derivatives and the fixed points.

The idea of the phase plane method is that the right hand sides of eqs. (34) define the local direction of derivatives, which is uniquely given by coordinates $(u, \mathbf{S})$ on the phase plane. Knowing this vector field, the solutions of the equations are simply integral curves obtained by following the vector field starting from any initial condition. A special role is played by the zeroes of this vector field, i.e., stable solutions or fixed points of the differential equations, and by nullclines, which are the lines along which one or the other derivative vanishes.

The nullclines of $u$ are the lines $\eta_{0}$, which corresponds to $u=0$, and $\eta_{*}$, which is $\mathbf{S}=c_{s}^{2}-3$. The nullcline $\eta_{0}$ happens to be an integral curve, since the vector field is everywhere tangent to this line. The nullclines of $\mathbf{S}$ are the hyperbolae $\mathbf{S}^{2}+\mathcal{B} \mathbf{S}\left[u-\left(1+c_{s}^{2}\right) / \mathcal{B}\right]=\mathcal{A}$. This has two branches, $\sigma_{-}$has $\mathbf{S}<0$ and is asymptotic to the $u$-axis at $u=-\infty, \sigma_{+}$has $\mathbf{S}>0$ and is asymptotic to the $u$-axis at $u=\infty$.

These two sets of nullclines cross at three fixed points $-P_{+}=\left(u_{+}, \mathbf{S}_{+}\right)$is the intersection of $\eta_{0}$ and $\sigma_{+}, P_{-}=$ $\left(u_{-}, \mathbf{S}_{-}\right)$, which is the intersection of $\eta_{0}$ and $\sigma_{-}$and $P_{*}=\left(u_{*}, \mathbf{S}_{*}\right)$ which lies on $\eta_{*}$ and $\sigma_{-}$, and

$$
\begin{aligned}
& u_{ \pm}=0, \quad \mathbf{S}_{ \pm}=\frac{1}{2}\left[1+c_{s}^{2} \pm \sqrt{4 \mathcal{A}+\left(1+c_{s}^{2}\right)^{2}}\right] \\
& u_{*}=\frac{1}{\mathcal{B}}\left[\frac{\mathcal{A}}{c_{s}^{2}-3}+4\right], \quad \mathbf{S}_{*}=c_{s}^{2}-3 .
\end{aligned}
$$

These features are shown in Figure 7

In a small interval around any fixed point $\left(u_{f}, \mathbf{S}_{f}\right)$ one can linearize the equations to get

$$
\partial_{\theta}\left(\begin{array}{c}
u-u_{f} \\
\mathbf{S}-\mathbf{S}_{f}
\end{array}\right)=\mathbf{M}\left(u_{f}, \mathbf{S}_{f}\right)\left(\begin{array}{c}
u-u_{f} \\
\mathbf{S}-\mathbf{S}_{f}
\end{array}\right), \quad \text { where } \quad \mathbf{M}(u, \mathbf{S})=\left(\begin{array}{cc}
\frac{1}{4}\left[\mathbf{S}+3-c_{s}^{2}\right] & u / 4 \\
-\mathcal{B} \mathbf{S} & -2 \mathbf{S}+\left(1+c_{s}^{2}\right)-\mathcal{B} u
\end{array}\right)
$$

At $P_{ \pm}$one of the off-diagonal components vanishes as a result of which one can write down the eigenvalues by inspection. $P_{+}$is a hyperbolic fixed point. $P_{-}$is a repulsive fixed point for a Boltzmann fluid, but changes into a hyperbolic point for large enough $\mathcal{A}$. One also finds that $P_{*}$ is a hyperbolic point for a Boltzmann fluid but changes into a repulsive fixed point for sufficiently large $\mathcal{A}$. The reason for this is not hard to understand. At $\mathcal{A}=4\left(3-c_{s}^{2}\right)$ the points $P_{-}$and $P_{*}$ are coincident and exchange character, leading to a saddle-node bifurcation at this value of $\mathcal{A}$. 

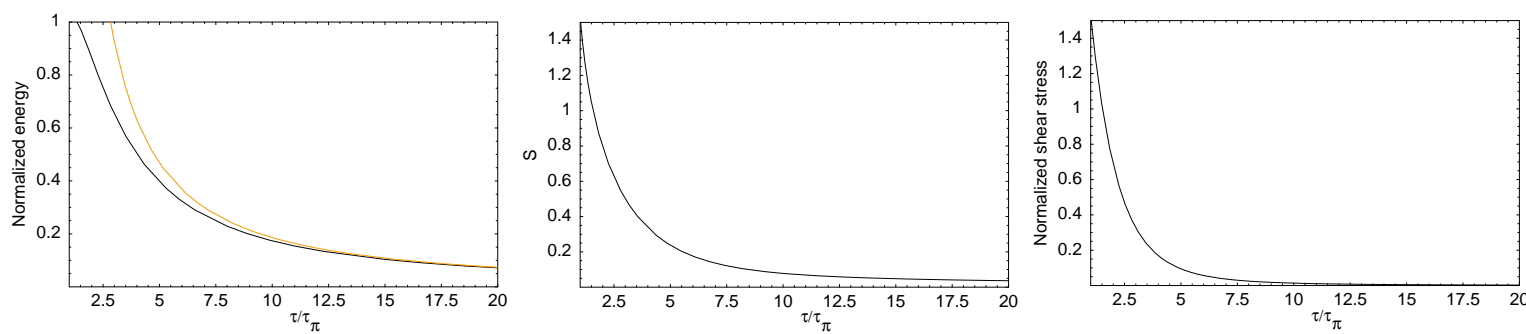

FIG. 8: (Color online) The solutions corresponding to the unstable manifold for the normalized energy, $\epsilon / \epsilon_{0}=u(\theta)^{4} \mathrm{e}^{-4 \theta}$, the quantity $\mathbf{S}$, and the normalized shear stress, $\mathbf{S} u(\theta)^{4} \mathrm{e}^{-4 \theta}$, plotted against $\tau / \tau_{\pi}$. In the figure for the normalized energy, the curve in yellow shows the Bjorken solution normalized to match the exact numerical solution at large $\tau / \tau_{\pi}$.

\section{The unstable manifold of $P_{+}$: a physically relevant solution}

We concentrate on the physical flows, i.e., those originating on the half-line $u=1$ and having $\mathbf{S} \geq 0$. Since the vector $\mathbf{V}$ points into the part of the plane bounded by $u=1$ and $\mathbf{S}=0$, one sees that physical flows remain in this portion of the phase plane. Also, since there are no fixed points in this part of the plane, one finds that flows cover the whole quadrant. Using Bendixson's theorems [39], one can prove the intuitively obvious fact that there are no periodic or quasi-periodic solutions starting from these initial conditions.

The main organizing element behind the physical flows is a special solution, $\mathcal{U}$, i.e., a curve in the phase plane, called the unstable manifold of $P_{+}$. This is the solution with initial conditions in the infinitesimal neighbourhood of $P_{+}$, the explicit numerical solution for which is exhibited in Figure 8 . Since $P_{+}$is a hyperbolic fixed point, all solutions starting from initial conditions above $\mathcal{U}$ are attracted to it from above, and those starting below it are attracted towards it from below. Clearly, then, an important element of the analysis is $\mathbf{S}_{\mathcal{U}}$, the point at which $\mathcal{U}$ intersects the line of physical initial conditions $u=1$.

Two varieties of stability in the solutions should be noted. At late times the flows are stable against changes in initial conditions, since they are always attracted to $\mathcal{U}$. One useful consequence is that fairly crude PDE solvers suffice to integrate eqs. (34). There is a deeper level of stability, called structural stability, arising from the fact that $P_{+}$does not collide with any of the other fixed points on changing the parameters $c_{s}^{2}, \mathcal{A}$, and $\mathcal{B}$. This means that for all values of these parameters, the nature of physical flows is determined by the corresponding solution for $\mathcal{U}$. The usefulness of structural stability is that extraction of parameters from data becomes particularly simple.

The first step to solving for the flows is to determine $\mathcal{U}$. In the neighbourhood of $P_{+}$it coincides with the eigenvector corresponding to the positive eigenvalue of $\mathbf{M}\left(u_{+}, \mathbf{S}_{+}\right)$. Using eqs. (35) 36), it is easy to see that this is the line $4 \mathcal{B} \mathbf{S}_{+} u=\left(1+5 c_{s}^{2}-9 \mathbf{S}_{+}\right)\left(\mathbf{S}-\mathbf{S}_{+}\right)$. Using $\mathcal{A}=8 / 27, \mathcal{B}=1$ and $c_{s}^{2}=1 / 3$ gives $\mathbf{S}_{\mathcal{U}}=0.975913$ in this linear approximation. The numerical continuation of the straight line is easy, since any initial condition close to $P_{+}$will quickly settle down to $\mathcal{U}$. Such a construction using the parameter values above gives $\mathbf{S}_{\mathcal{U}}=1.02545$, showing that the slope of $\mathcal{U}$ decreases marginally beyond the linear analysis.

Solving for the distant part of $\mathcal{U}$ is equivalent to finding the physical flows at long times. For this, we examine $u \gg 1$. From the phase space analysis we see that $\mathbf{S}$ decreases as $u$ increases when both are in the physical region. Hence, in the second of eqs. (34) we can neglect the term in $\mathbf{S}^{2}$ with respect to the others. Also, the term in $\left(1+c_{s}^{2}\right) \mathbf{S}$ can be neglected with respect to the remaining two terms. Then the system of coupled equations to be solved is-

$$
\partial_{\theta}\left(\begin{array}{c}
u \\
\mathbf{S}
\end{array}\right)=\left(\begin{array}{c}
u\left[\mathbf{S}+3-c_{s}^{2}\right] / 4 \\
\mathcal{A}-\mathcal{B} u \mathbf{S}
\end{array}\right)
$$

Initial conditions $u_{0}$ and $\mathbf{S}_{0}$ are imposed at $\theta=0$ in order to match this asymptotic solution with the full solution. Unfortunately, even this equation is too complicated for an analytic solution.

We do not need all solutions of eq. (37), as it happens. Since $\varphi<4 / 3$ for hydrodynamics to apply, we must have $\mathbf{S}<4 / 3 \chi=\mathcal{A}$. For the Boltzmann fluid, then, $\mathbf{S}<8 / 27$. Of course, this does not restrict all physical trajectories to approach $\mathcal{U}$ from below; trajectories can approach this curve from above, but they correspond to a different $\mathcal{B}$. An explicit asymptotic form is easy to write down when $\mathbf{S} \ll 3$. Then $\mathbf{S}$ can be neglected in the equation for $u$, and one gets-

$$
u(\tau)=u_{0}\left(\frac{\tau}{\tau_{0}}\right)^{\left(3-c_{s}^{2}\right) / 4} \text { implying } \epsilon(\tau)=\epsilon_{0}\left(\frac{\tau_{0}}{\tau}\right)^{1+c_{s}^{2}}
$$




$$
\mathbf{S}(\tau)=\exp \left[-\frac{4 \mathcal{B} u(\tau)}{3-c_{s}^{2}}\right]\left\{\mathbf{S}_{0}+\frac{4 \mathcal{A}}{3-c_{s}^{2}} \operatorname{Ei}\left(\frac{4 \mathcal{B} u(\tau)}{3-c_{s}^{2}}\right)\right\}
$$

Since these forms are asymptotically valid, the constants $\mathbf{S}_{0}, u_{0}$ and $\epsilon_{0}$ are free parameters which ensure that the asymptotic solution matches the exact solution at large $\tau$. The forms above are not to be extrapolated down to small $\tau$. In this limit one recovers Bjorken scaling, i.e., the boost-invariant solution of the ideal gas. Not only is this a satisfactory mathematical result, it could also be of physical relevance, if late freezeout occurs. For later reference we note that at late times one has

$$
\mathbf{S}(\tau)=\frac{\mathcal{A}}{\mathcal{B} u_{0}}\left(\frac{\tau_{0}}{\tau}\right)^{\left(3-c_{s}^{2}\right) / 4}
$$

using the asymptotic expansion of the exponential integral. The solution illustrated in Figure 8 shows that, as a result of viscosity, the expansion is slower than Bjorken, so that the energy density is diluted less rapidly. As a result, the initial energy density, as inferred from an observed final energy density, is very much smaller than the Bjorken estimate.

From the phase plane analysis, it is clear that physical flows with initial conditions lying below $\mathbf{S}_{\mathcal{U}}$ are attracted to $\mathcal{U}$ from below. For such solutions $\mathbf{S}$ increases initially before decreasing. Such solutions have been exhibited in [11, 14]. Other initial conditions for physical flows give rise to monotonically decreasing solutions for $\mathbf{S}$. The solution exhibited in Figure 8 is the critical solution, $\mathcal{U}$, which separates these two types of solutions. The long time behaviour of all solutions is arbitrarily close to $\mathcal{U}$.

\section{The ELNS approximation}

As discussed before, the ELNS limit of the equations can be obtained by dropping the term in the derivative of $\pi_{V}$, and then introducing an arbitrary scale of time called $\tau_{\pi}$. In this approximation, the equations for the Boltzmann fluid become

$$
u_{\theta}=\frac{1}{4} u\left[\mathbf{S}+3-c_{s}^{2}\right], \quad \text { and } \quad \mathcal{B} u \mathbf{S}=\mathcal{A} .
$$

Substituting the expression for $\mathbf{S}$ obtained from the second equation into the first, the equation can be easily integrated with the initial condition $u(\theta=0)=1$, to give

$$
u(\theta)=\mathrm{e}^{\theta(1-B / 4)} f(\theta), \quad \frac{\epsilon(\theta)}{\epsilon_{0}}=\mathrm{e}^{-B \theta} f^{4}(\theta), \quad \text { where } \quad f(\theta)=1+\frac{\mathcal{A}}{\mathcal{B}(4-B)}\left(1-\mathrm{e}^{(B / 4-1) \theta}\right),
$$

and $B=1+c_{s}^{2}$. This is in the form of the Bjorken solution modified by a factor which goes to a constant at large $\theta$. As before, the ELNS solution matches the IS solution at times much larger than the intrinsic time scale $\tau_{\pi}$.

\section{Entropy production}

For a Boltzmann fluid the entropy density is proportional to $\sigma=u^{3} \exp (-3 \theta)$. One can then manipulate eq. (34) into the form

$$
\frac{d \sigma}{d \theta}=\frac{3}{4} \sigma\left(\mathbf{S}-\frac{4}{3}\right)
$$

where we have used the value $c_{s}^{2}=1 / 3$. Since $\mathbf{S}$ decreases with time, it is clear that at sufficiently late time the factor $\mathbf{S}-4 / 3$ becomes negative, and hence the entropy density must decrease. The total entropy scales as $\Sigma=\sigma \exp \theta$, since the spatial volume element picks up a scale factor of $\tau$ from the metric. For this quantity we find the equation

$$
\frac{d \Sigma}{d \theta}=\frac{3}{4} \Sigma \mathbf{S},
$$

which is positive definite since the factors on the right hand side are all positive. Hence the total entropy must increase. Using the expression for $\mathbf{S}$ in eq. (38), one finds that $\ln \Sigma$ can be written in terms of Meijer-G functions. Using instead the asymptotic expansion of $\mathbf{S}$ in eq. (39), one obtains the simpler long-time limit

$$
\Sigma(\tau)=\Sigma\left(\tau_{0}\right) \exp \left[\frac{9 \mathcal{A}}{8 \mathcal{B} u_{0}}\left\{1-\left(\frac{\tau_{0}}{\tau}\right)^{2 / 3}\right\}\right] .
$$

In contrast to the simple fluid, where the entropy increases without bound, the entropy of an expanding Boltzmann fluid reaches a finite limit. The solution is shown in Figure 9 


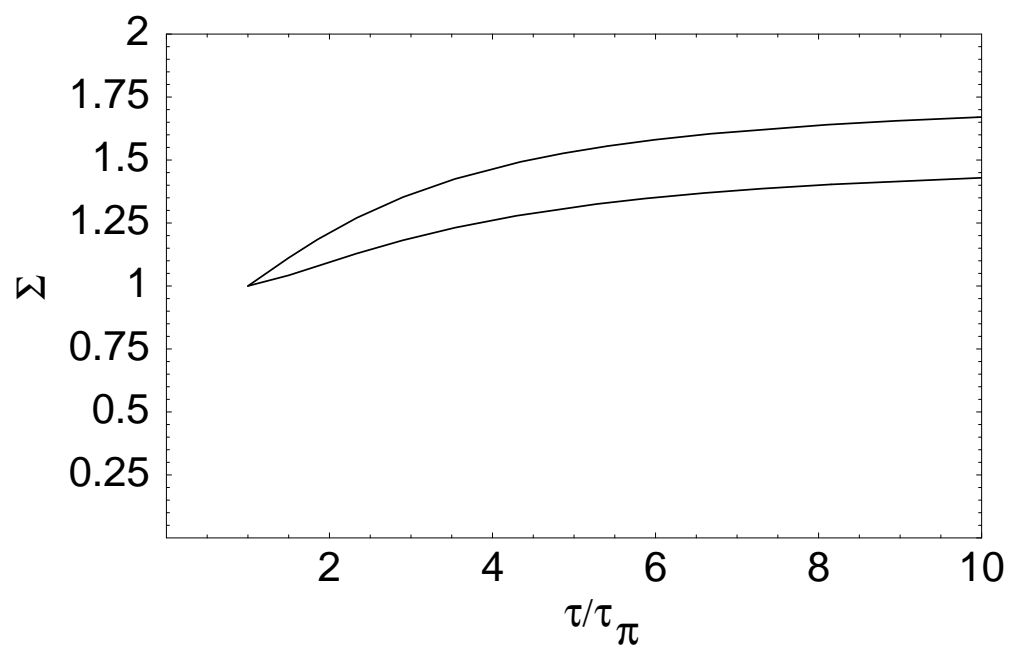

FIG. 9: The normalized entropy $\Sigma$ as a function of $\tau / \tau_{\pi}$. Note the saturation at large $\tau$. The numerical solution is for $\mathcal{A}=8 / 27$ and $\mathcal{B}=1$. The upper curve is for $\mathbf{S}^{0}(0)=8 / 27$ and the lower for $\mathbf{S}^{0}(0)=2 / 27$.

\section{B. Sound waves}
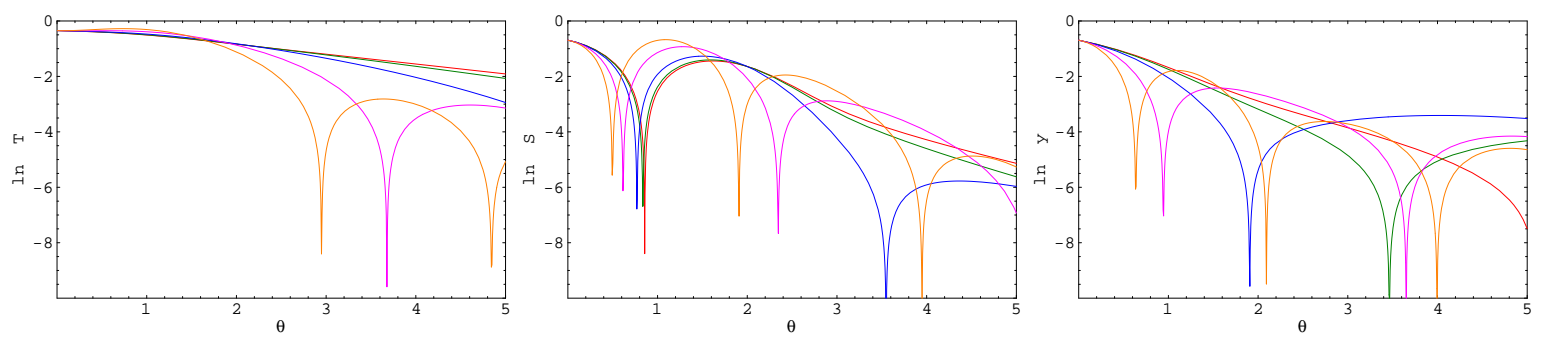

FIG. 10: (Color online) Solutions of the linearized equations for fluctuations around the scaling solution in a Boltzmann fluid. The normalized temperature, $T$, the quantity, $\mathbf{S}$, and $y$ are shown for $k=1 / 4$ (red), $1 / 2$ (green), 1 (blue), 2 (purple) and 3 (orange) for $\mathbf{S}^{0}(0)=8 / 27$. The other initial conditions are $u^{1}(0)=1 / \sqrt{2}, \mathbf{S}^{1}(0)=y^{1}(0)=1 / 2$.

The linearized equations for fluctuations in a Boltzmann fluid are

$$
\begin{aligned}
u_{\theta}^{1} & =\frac{1}{4}\left(3-c_{s}^{2}+\mathbf{S}^{0}\right) u^{1}-\frac{1}{4}\left(B-\mathbf{S}^{0}\right) u^{0} y_{\eta}^{1}+\frac{1}{4} u^{0} \mathbf{S}^{1}, \\
y_{\theta}^{1} & =\frac{4\left(c_{s}^{2}-\mathbf{S}^{0}\right)}{u^{0}\left(\mathbf{S}^{0}-B\right)} u_{\eta}^{1}+\frac{B\left(1-c_{s}^{2}\right)-\mathcal{A}+\mathbf{S}^{0}\left(\mathcal{B} u^{0}-1+c_{s}^{2}\right)}{\mathbf{S}^{0}-B} y^{1}-\frac{1}{\mathbf{S}^{0}-B} \mathbf{S}_{\eta}^{1}, \\
\mathbf{S}_{\theta}^{1} & =-\mathcal{B} \mathbf{S}^{0} u^{1}-\left[\left(\mathbf{S}^{0}\right)^{2}-B \mathbf{S}^{0}-\mathcal{A}\right] y_{\eta}^{1}-\left(\mathcal{B} u^{0}+2 \mathbf{S}^{0}-B\right) \mathbf{S}^{1},
\end{aligned}
$$

where we have made the expansion $f(\theta, \eta)=f^{0}(\theta)+\Delta f^{1}(\theta, \eta)$ where $f$ is any of $u, y$ and $\mathbf{S}$. As before, $y^{0}=0$, and $u^{0}$ and $\mathbf{S}^{0}$ are obtained as the solution of eqs. (34), and we can Fourier transform in $\eta$ to examine the evolution of each mode, $k$. Explicit solutions for $u^{0}$ and $\mathbf{S}^{0}$ can be written only in the long-time limit, when these tend to the unstable manifold $\mathcal{U}$. However, some physically interesting phenomena are likely to occur before this time. Hence, the method of choice is to numerically solve eqs. (34, 45) together for the five variables at the leading and first order in $\Delta$. The initial conditions for $u^{0}$ and $\mathbf{S}^{0}$ have been discussed before. Those for $u^{1}, y^{1}$ and $\mathbf{S}^{1}$ can be chosen to lie between -1 and 1 in order for $\Delta$ to give the right order of the magnitude of fluctuations.

The results obtained using $c_{s}^{2}=1 / 3, \mathcal{A}=8 / 27, \mathcal{B}=1$ are shown in Figure 10. For large values of $k$, there are quasi-periodic solutions. For smaller values of $k$ the solutions are overdamped. The critical value, $k_{0}$, which separates damped and oscillatory solutions depends on $\mathbf{S}^{0}(0)$. The solutions do not change qualitatively if $\mathcal{B}$ is changed by an order of magnitude. From the solutions displayed, it is clear that a damping exponent can be extracted. 

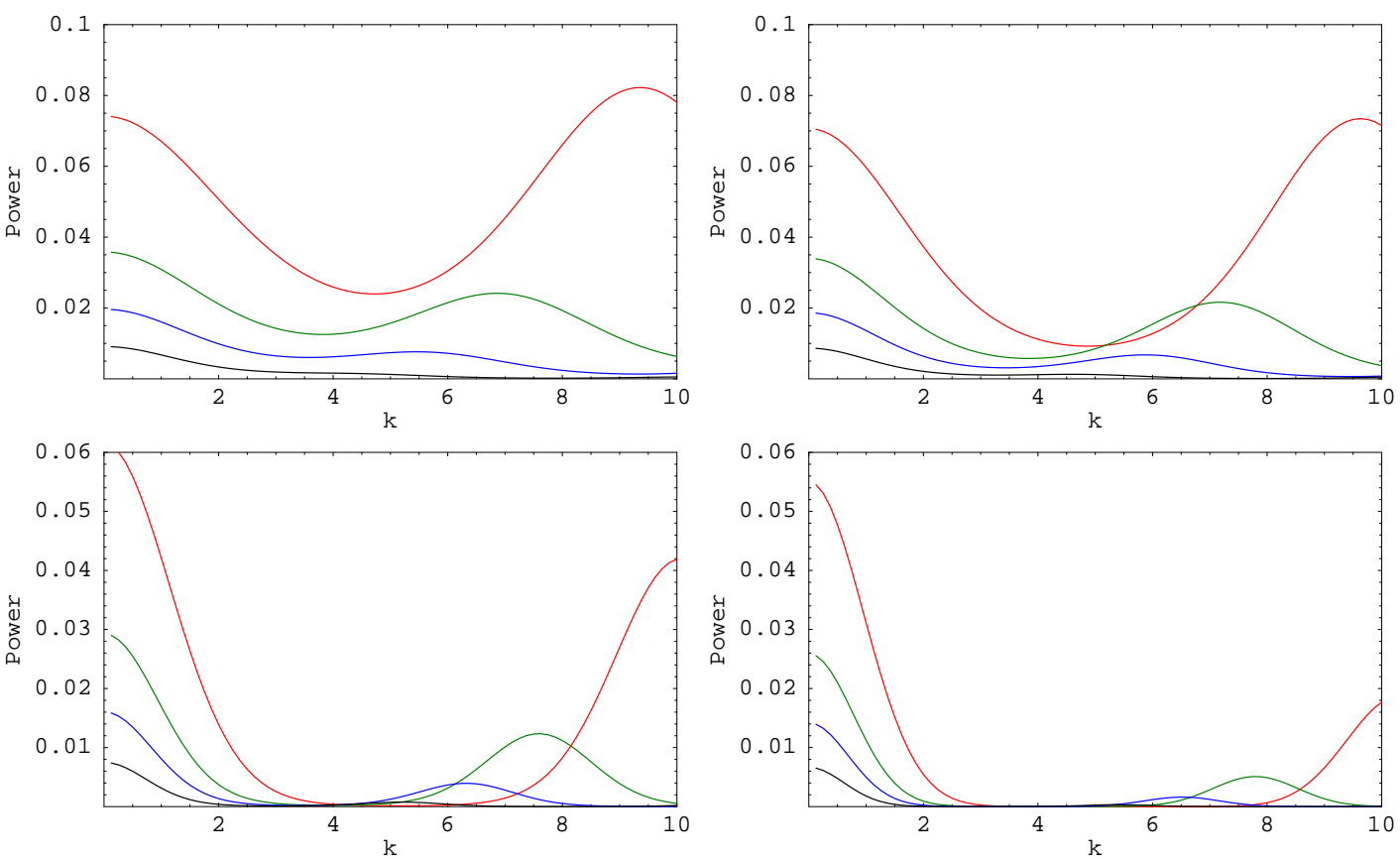

FIG. 11: (Color online) The power spectrum of fluctuations after evolution from an initially flat spectrum at $\theta=0.8$ (red), 1 (green), 1.25 (blue) and 1.5 (black). The numerical solution is obtained for $c_{s}^{2}=1 / 3, \mathcal{B}=1, \mathcal{A}=8 / 27$ for four different values of $\mathbf{S}^{0}(0)$. The other initial conditions are $u^{1}(0)=-0.95, y^{1}(0)=-0.5$ and $\mathbf{S}^{1}(0)=0.3$. Note the difference in the scales.

A quantity which encapsulates the physics, and is perhaps better suited to making a connection with experiments is the power spectrum. In Figure 11 we display the evolution of the power spectrum starting from an initially flat spectrum. Qualitatively, the behaviour is reminiscent of the simple fluid examined earlier. Evolution produces peaks in the power spectrum. The positions of these peaks evolve with time- moving to smaller $k$ due to the redshifting discussed earlier. The position and magnitude of the peaks depend very strongly on initial conditions and $c_{s}$. For the relation between the power spectrum studied here and the correlation function in [26], see Section III.

The connection with ELNS hydrodynamics is made, as before, by dropping the term in the time derivative of $\pi_{V}$. As we have mentioned before, this results in the hyperbolic equations turning into a parabolic set. As a result, fluctuations are diffusively damped, and do not turn into propagating sound waves. This is seen in numerical solutions, and will be dealt with more completely in the next section.

\section{A CONFORMAL FLUID}

As discussed in Section II.A, a conformal fluid differs from a Boltzmann fluid only in the $T$-independent value $\chi=\epsilon \tau_{\pi} / \eta_{V}=3 \pi a$, where $a=\tau_{\pi} T$. The hydrodynamics of such a conformal fluid can then be taken over from that of the Boltzmann fluid with the simple replacement $\mathcal{A}=4 / 3 \chi=4 / 9 \pi a$. In a strongly interacting fluid one might expect $a=\tau_{\pi} T$ to be small, and hence $\mathcal{A}$ to be large. The separation into boost-invariant and fluctuation equations and the analysis of each, is very similar to the details presented in Section V. For this reason, we do not repeat the material here, but only point out the differences.

The boost-invariant flows follow eqs. (34). As discussed in Section V.A, the global structure of the flows is determined by the three fixed points $P_{*}, P_{-}$and $P_{+}$. When $\mathcal{A}$ is large enough, the roles of the fixed points $P_{-}$and $P_{*}$ are interchanged. However, physical flows are governed by the unstable manifold of $P_{+}$and its behaviour remains unchanged. In particular, one may take over the expressions for the long-time behaviour of the unstable manifold.

The boost-invariant flows, starting from the same initial conditions, as $\chi$ changes, are shown in Figure 12, The late-time behaviour of the trajectories in $\varphi^{0}=\chi \mathbf{S}^{0}$ and $u^{0}$ is independent of the value of $\mathcal{A}$. This is clear by using eqs. (39) to write

$$
\varphi^{0}=\frac{\mathcal{A} \chi}{\mathcal{B} u^{0}}=\frac{4}{3 \mathcal{B} u^{0}}
$$




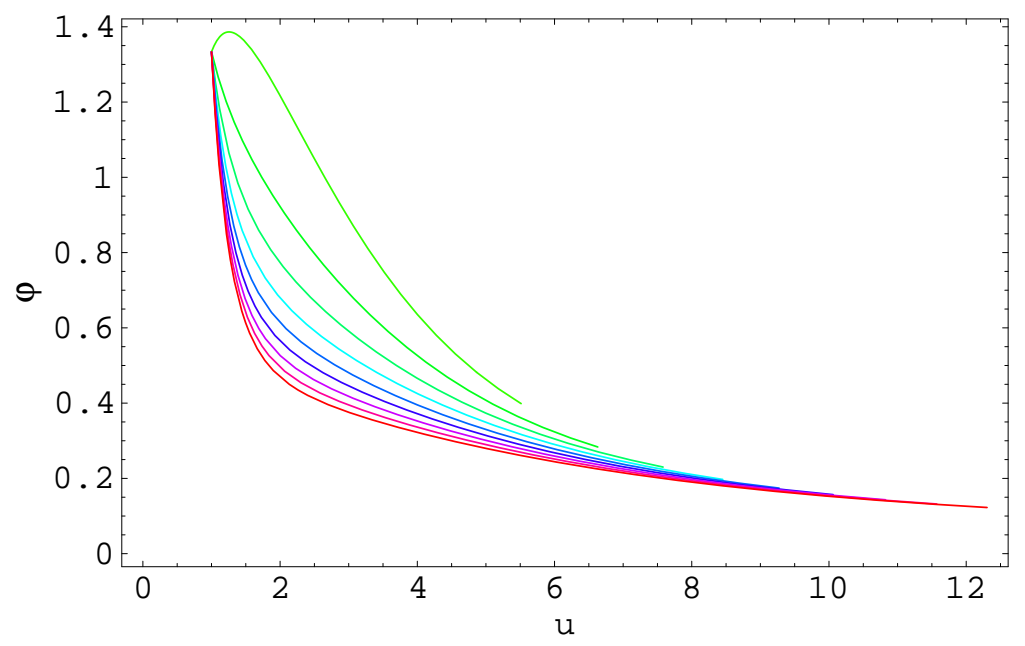

FIG. 12: (Color online) Boost-invariant flow for conformal fluids in the plane of $\varphi^{0}$ and $u^{0}$, starting from the same initial conditions, with $\mathcal{B}=1$, but with varying $\chi$, i.e., $\mathcal{A}$. As $\mathcal{A}$ increases from 1 to 9 in steps of 1 ( $\mathcal{A}$ increases from top to bottom), the long-time behaviour remains universal, but is approached faster.

This universality is a consequence of the structural stability of the hydrodynamic equations for this class of fluids. From the figure one can also see that for $\mathcal{A} \approx 1, \varphi^{0}$ increases before decreasing. Thermalization can be said to occur only when the solution enters the physical domain $\varphi^{0}<4 / 3$ for the last time.
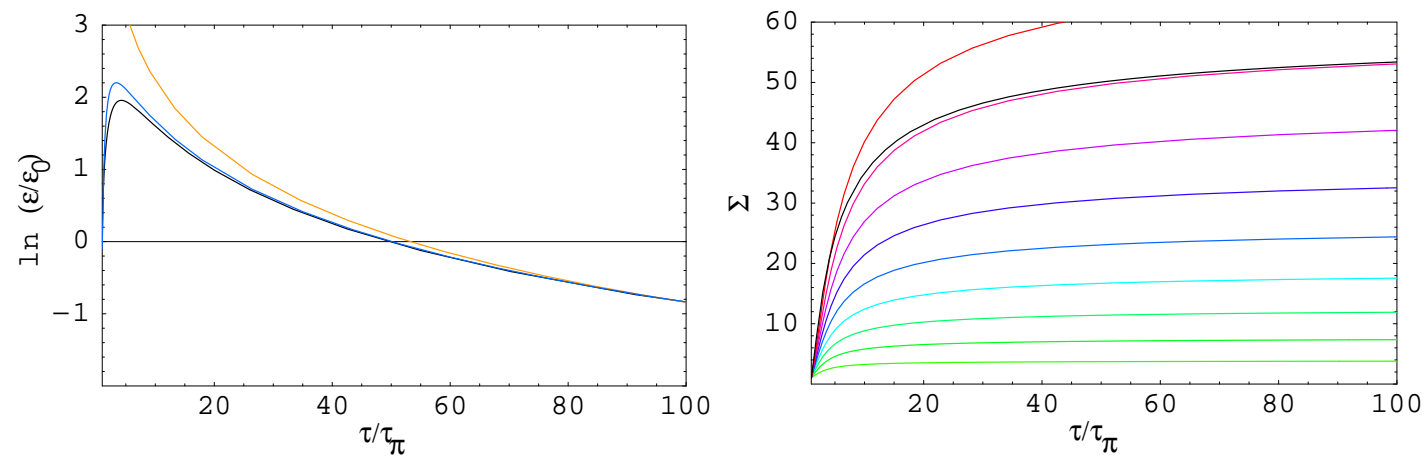

FIG. 13: (Color online) Exact numerical solutions for (a) the energy density and (b) total entropy along the unstable manifold for conformal fluids. In (a) the line in black shows the exact solution for $\mathcal{A}=8$, the line in gold is a Bjorken solution matched to it asymptotically, and the line in blue is the corresponding solution in ELNS hydrodynamics matched asymptotically. In (b) the coloured lines show the time-evolution of $\Sigma$ as $\mathcal{A}$ varies from 1 to 9 in steps of 1 , the larger the $\mathcal{A}$, the higher the curve. The line in black is the prediction from the ELNS solution matched to the energy density.

From eq. (44) it is clear that for all conformal fluids the amount of entropy generated during the flow, proportional to $\Sigma$, has a finite upper bound. However, this bound increases exponentially with $\mathcal{A}$, starting from the initial value $\Sigma\left(\tau_{0}\right)=1$, independent of $\mathcal{A}$. Note that this means that at fixed value of $\eta_{V} / s$, the maximum entropy production has exponential dependence on the inverse relaxation time, $1 / \tau_{\pi}$.

Since the longitudinal flow can be continued to times of order $\tau_{T}$, and $\tau_{T}$ is independent of any material property other than $c_{s}$, for conformal fluids, the ratio $\tau_{T} / \tau_{\pi}$ could become large as $\tau_{\pi}$ decreases. One might then expect that ELNS evolution should suffice to describe the system. Figure 13 illustrates several interesting points. First, the late time evolution of the energy density can be approximated by an appropriately tuned Bjorken solution, the tuning parameter being the initial energy density. However, as shown in the figure, this gives a gross over-estimate of the energy density; in the example, by more than two orders of magnitude. Second, a solution of ELNS hydrodynamics can be tuned to reproduce the energy density at late times. Once this tuning is performed, it also reproduces the total entropy production at late times, and hence furnishes a far superior description of the flow than the Bjorken solution could. Note, however, that the ELNS solution has larger entropy production than the true IS solution at 

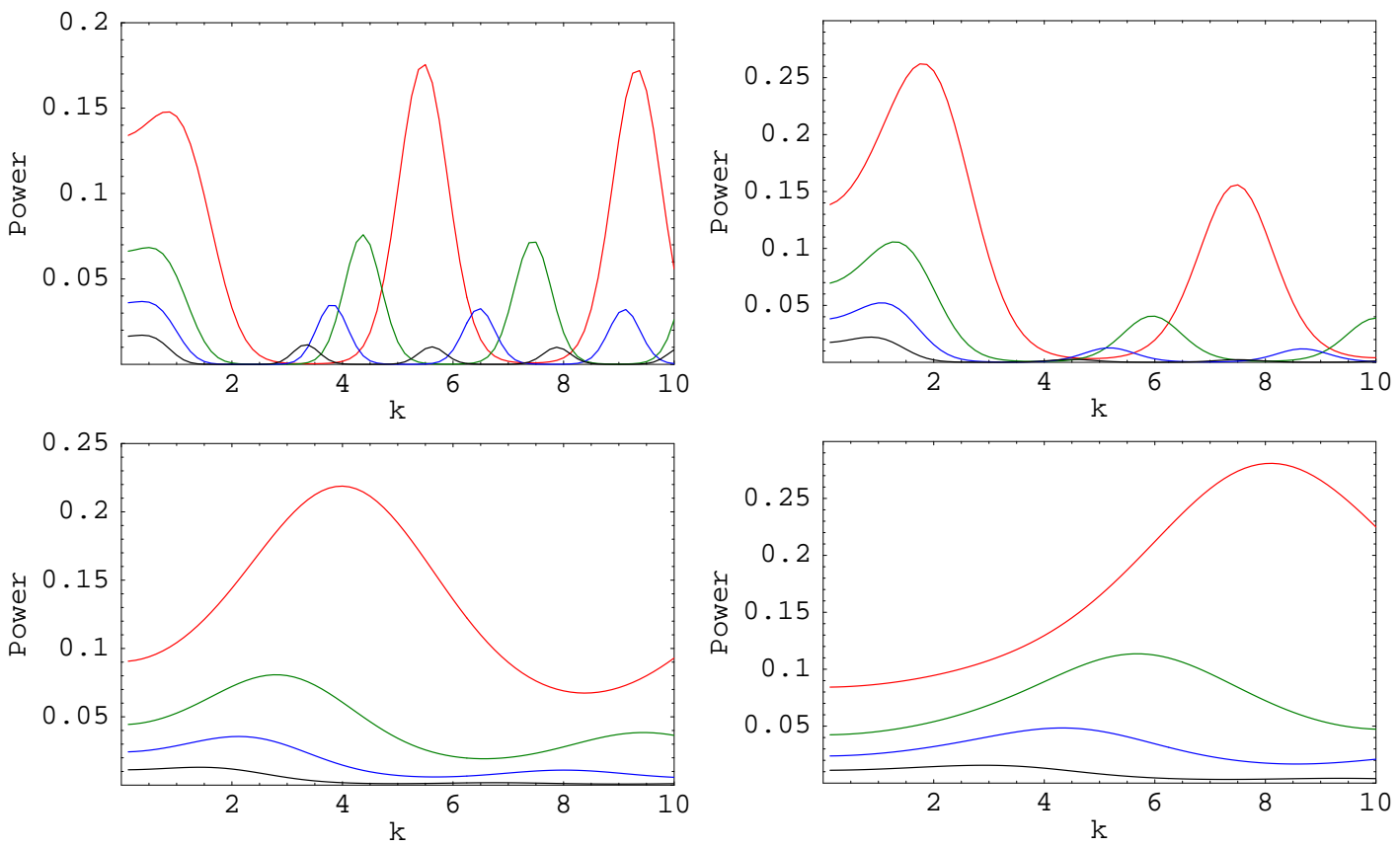

FIG. 14: (Color online) The power spectrum for the energy density, at $\theta=0.8$ (red), 1.05 (green), 1.25 (blue) and 1.5 (black), starting from a constant unit distribution with initial conditions $\mathbf{S}^{0}(0)=\min (1, \mathcal{A}), u^{1}(0)=-0.95, y^{1}(0)=-0.55$ and $\mathbf{S}^{1}(0)=0.30$ for all $k$. The successive figures are for (a) $\mathcal{A}=2$, (b) $\mathcal{A}=1$, (c) $\mathcal{A}=1 / 2$, (d) $\mathcal{A}=1 / 3$.

initial times and that there are quantitative lacunae in this approximation even at $\tau=40 \tau_{\pi}$. With lower $\mathcal{A}$, we have seen that these discrepancies are larger. Finally, we note that the maximum entropy production occurs in the very early stages of the flow, and probes of this stage of the expansion would best discriminate between different values of $\tau_{\pi}$. A different approach to extracting $\tau_{\pi}$ is advocated in [17].

A possible discriminant between ELNS and IS hydrodynamics is the fate of fluctuations around the scaling solution. An analysis of fluctuations can be performed numerically using eqs. (45), as before. At small $k$ all solutions are overdamped, as can be seen by investigating the $k \rightarrow 0$ limit, as before. At large $k$ the fluctuations develop into damped propagating waves. The evolution of the power spectrum of the fluctuations in energy density, starting from a uniform spectrum is shown in Figure 14. For generic initial conditions, increasing $\mathcal{A}$, i.e., decreasing $\tau_{\pi}$, seems to damp fluctuations faster. However, the equations become stiff for $\mathcal{A}>2$ and the numerical solutions are hard to extract for the interesting case of $N=4$ SYM theory which yields $\mathcal{A}=8.69$.

However, in that case, we can take another approach. The asymptotic solutions exhibited in eqs. (38, 39) are reliable for large $\theta$. One can investigate the fate of fluctuations around the scaling solution at late times by inserting the asymptotic formulæ into eqs. (45). The asymptotic solutions can be written as $u^{0}=u_{0} \exp (p \theta)$ and $\mathbf{S}^{0}=$ $\left(\mathcal{A} / \mathcal{B} u_{0}\right) \exp (-p \theta)$, where $p=1-B / 4$. We expand eqs. (45) in powers of $z=\exp (p \theta)$, and retain all non-negative powers of $z$ in the equations. This gives

$$
\begin{aligned}
& \partial_{\theta}\left(\begin{array}{l}
u^{1} \\
y^{1} \\
\mathbf{S}^{1}
\end{array}\right)=M\left(\begin{array}{l}
u^{1} \\
y^{1} \\
\mathbf{S}^{1}
\end{array}\right), \quad \text { where } M=i k M_{1}+M_{0}, \\
& M_{1}=\left(\begin{array}{ccc}
0 & -\frac{B u_{0}}{4} \mathrm{e}^{p \theta} & 0 \\
0 & 0 & \frac{1}{B} \\
0 & \mathcal{A} & 0
\end{array}\right), \quad M_{0}=\left(\begin{array}{ccc}
p & 0 & \frac{u_{0}}{4} \mathrm{e}^{p \theta} \\
0 & -\left(1-c_{s}^{2}\right) & 0 \\
0 & 0 & -u_{0} \mathcal{B} \mathrm{e}^{p \theta}
\end{array}\right) .
\end{aligned}
$$

Note that the set $y^{1}$ and $\mathbf{S}^{1}$ can be solved independently of $u^{1}$, and this last variable is then driven by the others.

For orientation, let us examine some analytical approximations first. As $k \rightarrow 0$, one may set $M=M_{0}$. The equations are then exactly solvable, and yield

$$
\mathbf{S}^{1}(\tau)=\mathbf{S}_{0}^{1} \exp \left[\frac{u_{0} \mathcal{B}}{p}\left\{1-\left(\frac{\tau}{\tau_{0}}\right)^{p}\right\}\right],
$$




$$
\begin{aligned}
& y^{1}(\tau)=y_{0}^{1}\left(\frac{\tau_{0}}{\tau}\right)^{1-c_{s}^{2}} \\
& u^{1}(\tau)=\left(\frac{\tau}{\tau_{0}}\right)^{p}\left[u_{0}^{1}-\frac{u_{0} \mathbf{S}_{0}^{1}}{4 p} \mathrm{e}^{\mathcal{B} u_{0} / p}\left\{\operatorname{Ei}\left(-\frac{\mathcal{B} u_{0}}{p}\right)-\operatorname{Ei}\left(-\frac{\mathcal{B} u_{0}}{p}\left(\frac{\tau}{\tau_{0}}\right)^{p}\right)\right\}\right] .
\end{aligned}
$$

All these expressions must be truncated at order $z^{0}$ by dropping all terms of order $1 / z$ or smaller, since the equations were obtained similarly. At large $\tau$, the temperature fluctuation, $u^{1} \tau_{0} / \tau$ decreases as $1 / \tau^{1-p}, y^{1}$ decreases as $1 / \tau^{1-c_{s}^{2}}$, and $\mathbf{S}^{1}$ decreases exponentially.
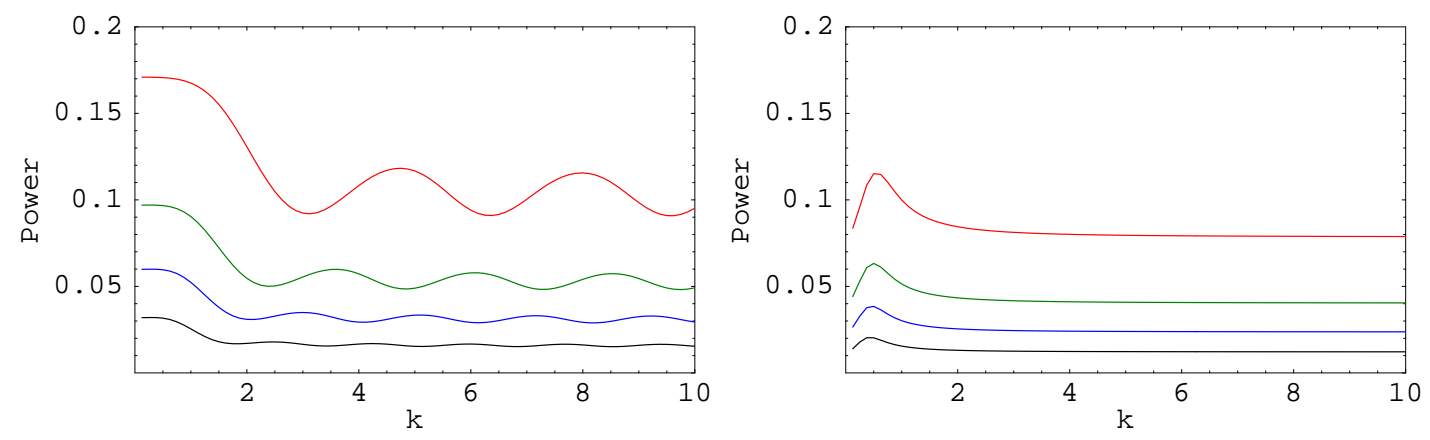

FIG. 15: (Color online) The late-time power spectrum for the energy density, at $\theta=0.8$ (red), 1.05 (green), 1.25 (blue) and 1.5 (black), starting from a constant unit distribution in (a) IS hydrodynamics with $\mathcal{A}=8$ and initial conditions $u^{1}(0)=-0.95$, $y^{1}(0)=0.95$ and $\mathbf{S}^{1}(0)=-0.75$ for all $k$ and (b) ELNS hydrodynamics with the same initial values of $u^{1}$ and $y^{1}$.

At large $k$, neglecting $M_{0}$, one finds that the solutions for $\mathbf{S}^{1}$ and $y^{1}$ are oscillatory with frequencies $i \omega= \pm i k \sqrt{\mathcal{A} / B}$. The solution for $u^{1}$ is entirely driven by $y_{1}$, and hence is oscillatory with a growing amplitude. The growth exponent precisely matches the growth exponent of $u^{0}$, hence $u^{1} / u^{0}$ is purely oscillatory in this approximation. Damping arises with the inclusion of $M_{0}$. Then the equations are not autonomous, and one could hesitate to treat the matrices $M_{1}$ and $M_{0}$ as time independent. However, by choosing $k$ to be large enough, one may be able to treat $\exp (p \theta)$ as a constant over many periods of oscillation. The simplest approach to computing the damping exponent then is to treat the problem in perturbation theory. This is best done, as before, by assuming that $i \omega=i k \lambda_{1}+\lambda_{0}+\mathcal{O}(1 / k)$, introducing this expansion into the characteristic equation for $M$ and then solving for $\lambda_{0,1}$. As expected, $\lambda_{1}= \pm \sqrt{(\mathcal{A} / B)}$ and 0 . The damping exponent is $-\lambda_{0}$. We find that $y^{1}$ and $\mathbf{S}^{1}$ have the common damping exponent $\left[1-c_{s}^{2}+u_{0} \mathrm{e}^{p \theta}\right] / 2$. The temperature fluctuations, $u^{1} / u^{0}$ are also damped.

The equations of ELNS hydrodynamics can be recovered from those of IS hydrodynamics by the method which we have explained at length earlier. In this case, the ELNS equations are obtained by first setting $\mathbf{S}^{0}=\mathcal{A} / \mathcal{B} u^{0}$ (see eq. 37), an approximation which is equivalent to the late-time solution in eq. (39), and reducing the last of eqs. (47) through the further identification $\mathbf{S}^{1}=\mathcal{A} y_{\eta}^{1} / \mathcal{B}$. Then the equation for $y^{1}$ turns into a diffusion equation, which has a completely different character from three equations of IS hydrodynamics which together give rise to wave propagation. The qualitative difference between the two kinds of hydrodynamics is very clear from the evolution of the power spectrum of temperature fluctuations, as shown in Figure 15. The propagation of damped sound waves in IS hydrodynamics is signalled by the formation of beats, and its absence in the case of ELNS signals the diffusive nature of the dynamics.

\section{CONCLUSIONS}

In this paper we examined freely expanding fluids using causal viscous hydrodynamics [19] in the longitudinal 1-dimensional approximation. We chose coordinates appropriate to a description of longitudinal flow, performed a tensor analysis and reduced the tensor equations of hydrodynamics to three scalar equations. The hydrodynamic modes are described by three scalars, the energy density, $\epsilon$, shear viscous part of the energy-momentum tensor, $\pi_{V}$, and a parametrization of the velocity, $y$. Details of this procedure are given in Appendices A and B.

Material properties expected of the QCD fluid were discussed in Section II.A, and three models of the fluid were put forward. We gave the name "simple fluid" to a model in which $c_{s}, \eta_{V}$ and $\tau_{\pi}$ are temperature independent. Other toy models, which go by the names of Boltzmann fluid and conformal fluids, have $c_{s}^{2}=1 / 3$ and $\chi=\epsilon \tau_{\pi} / \eta_{V}$, both independent of the temperature. Different such fluids are distinguished by the value of $\chi$. For the same value of 
$\eta_{V} / s$, one can have any value for $\chi$; large values of the latter corresponding to gaseous fluids and small values to more liquid-like behaviour.

In Section II.B we performed an analysis of the symmetries of the hydrodynamic equations and extracted dimensionless scaling variables which are combinations of the hydrodynamic variables and material properties. These express laws of physical similarity, and can be related, in the non-relativistic limit, to the familiar dimensionless variables, i.e., the Reynolds number, Re, the Mach number, $M$, and the Knudsen number, $K$. For Boltzmann and conformal fluids, the condition that $\chi$ does not depend on the temperature implies the combination $K \operatorname{Re} / M$ is constant. We discussed how IS hydrodynamics provides a self-consistent description of thermalization.

In scaling flow of an ideal fluid, the initial energy density entirely goes into driving the expansion. This leads to the Bjorken expansion with its characteristic power law behaviour, $\epsilon \propto 1 / \tau^{B}$ (where $B=1+c_{s}^{2}$ ). In contrast, in a viscous fluid, some part of this energy density is dissipated, leading to entropy production. Viscous effects are sufficiently strong in the simple fluid that the power law is modified to $\epsilon \simeq 1 / \tau$ asymptotically (section IV.A). As a result, the total entropy of the system increases without bound (section IV.A.2); we find $\Sigma \propto \tau^{1 / 4}$ asymptotically.

We made a phase plane analysis of flows for conformal fluids, including the Boltzmann fluid (see Section V.A.1). Our analysis shows that the long-time behaviour of any physical flow is arbitrarily close to a special solution of the hydrodynamic equations which we call the unstable manifold, $\mathcal{U}$. We investigated this solution in detail (see Sections V.A.2 and V.A.4), in particular, the approach to the Bjorken solution and rates of entropy production.

In the Boltzmann fluid, Bjorken scaling is recovered asymptotically (Section V.A.2). However, the initially slower expansion means that the energy density is diluted slower; Bjorken estimates of the initial energy density, given the final, are therefore over-estimates. The total entropy is saturated reasonably early (section V.A.4). Such behaviour also holds for conformal fluids (section VI). However, for fixed $\eta_{V} / s$, the saturation value for the entropy depends exponentially on the relaxation time $\tau_{\pi}$. The rate of growth of the entropy does not depend uniquely on $\eta_{V} / s$, since the parameter $\tau_{\pi}$ also plays a role.

When the relaxation time for the shear stress, $\tau_{\pi}$ is very small in units of $1 / T$ (i.e., the fluid behaves like a liquid), then the late time solution for a conformal fluid is reasonably well approximated in ELNS hydrodynamics by tuning a free parameter. This free parameter is equivalent to the initial energy density (section VI). After performing such a fit, the entropy density can also be fitted provided an appropriate unit of time is chosen. Hence, the extraction of initial conditions and material properties from observed final data remain as equivalently hard problems in ELNS and IS hydrodynamics. Furthermore, the initial conditions inferred from a given final energy density in the two kinds of dynamics differ by a large factor. Hence, in making inferences about the system produced in heavy-ion collisions, one must be careful to use the right type of hydrodynamics.

For an ideal fluid, the fluctuations around the scaling solution are overdamped for spatial Fourier modes $k<k_{0}=$ $\left(1-c_{s}^{2}\right) / 2 c_{s}$. For larger $k$, the fluctuations become damped sound waves. The energy density in the fluctuations is damped as a power law in $\tau$ (section III). These qualitative features persist in IS hydrodynamics of viscous fluids (sections IV.B, V.B and VI). When $k$ is small enough, the solutions are overdamped. At larger $k$ damped sound waves are obtained. The scaling solutions are therefore stable against small fluctuations. The power spectra of fluctuations contain interesting structure, which, if observable, could give information on fluid properties or initial conditions.

Although the scaling solutions obtained using IS hydrodynamics can be well approximated at asymptotically late times by the solutions of ELNS hydrodynamics (sections IV.A.1, V.A.3, VI), fluctuations behave completely differently in these two kinds of dynamics. In the latter, there are no sound modes: all fluctuations are diffusively damped. In IS dynamics, however, modes with large enough $k$ give rise to true sound waves. These two kinds of behaviour are easy enough to distinguish through power spectra of the energy density (Figures 14, 15). Similar effects are also to be seen in the fluctuations of $y$. Note the rather precise analogy of acoustically produced peaks in the power spectrum of the temperature fluctuations in heavy-ion collisions with those in the blackbody radiation in the early universe.

One object of great modern interest in heavy-ion collisions has been the coupling between hard and soft particles, for example jet-quenching. An effective theory description of this is to treat the soft particles through fluid dynamics and describe the hard particle as forcing a shock wave in this medium. The behaviour of acoustics is an important ingredient in such an analysis. In view of the difference between ELNS and IS hydrodynamics in their treatment of fluctuations, they could potentially give rise to different predictions for such observables.

The purpose of this paper was to explore the kind of phenomena that arise in IS causal viscous hydrodynamics, and find qualitative features which are different from either ELNS hydrodynamics or ideal gases. The changes in the scaling solution are features which distinguish IS hydrodynamics from ideal gases. The propagation of fluctuations around these solutions as sound waves gives a qualitative distinction between IS and ELNS hydrodynamics, since these modes are diffusively damped in the latter case, and do not propagate.

Comparison with data, and fits to initial conditions and material properties are outside the scope of this paper. These questions require a proper treatment of radial and anisotropic flows, as well as the incorporation of bulk viscosity (due to its importance near and below $T_{c}$ ). These questions are left to the future. 


\section{APPENDIX A: TENSOR DECOMPOSITIONS}

\begin{tabular}{||c|c|cccc||}
\hline & $g_{\mu \nu}$ & $L_{\mu}^{\lambda}$ & $V_{\mu}^{\lambda}$ & $\Sigma_{\mu}^{\lambda}$ & $A_{\mu}^{\lambda}$ \\
\hline$L^{\mu \nu}$ & 1 & $L^{\nu \lambda}$ & 0 & 0 & $\frac{1}{2}\left(A^{\nu \lambda}+\mathcal{A}^{\nu \lambda}\right)$ \\
$V^{\mu \nu}$ & 1 & & $V^{\nu \lambda}$ & 0 & $\frac{1}{2}\left(A^{\nu \lambda}-\mathcal{A}^{\nu \lambda}\right)$ \\
$\Sigma^{\mu \nu}$ & 2 & & & $\Sigma^{\nu \lambda}$ & 0 \\
$A^{\mu \nu}$ & 0 & & & & $-L^{\nu \lambda}-V^{\nu \lambda}$ \\
\hline
\end{tabular}

TABLE I: Contractions of the symmetric basis tensors. The entries are the contractions of the tensors in the row and column. The contraction in the first column is the trace.

In longitudinal flow there are only two vectors intrinsic to the problem - the timelike $u$ and the spacelike $v$. One could construct two more spacelike vectors to complete a tetrad, but since these vectors are completely arbitrary, no physics can depend on them. In order to express the rank-two tensors which enter the hydrodynamic equations, we can only use combinations of $u$ and $v$, and the metric tensor.

The symmetric rank-two tensors can be chosen to be the projections $L^{\mu \nu}=u^{\mu} u^{\nu}, V^{\mu \nu}=-v^{\mu} v^{\nu}$, and $\Sigma^{\mu \nu}=$ $g^{\mu \nu}-L^{\mu \nu}-V^{\mu \nu}$. In addition there is the traceless symmetric tensor $A^{\mu \nu}=u^{\mu} v^{\nu}+u^{\nu} v^{\mu}$, and the antisymmetric tensor $\mathcal{A}^{\mu \nu}=u^{\mu} v^{\nu}-u^{\nu} v^{\mu}$. The double contraction of the antisymmetric and any of the four symmetric tensors vanishes. For notational convenience we also define the spacelike projection $\Delta^{\mu \nu}=g^{\mu \nu}-L^{\mu \nu}=\Sigma^{\mu \nu}+V^{\mu \nu}$. Contractions of this with the other tensors can be worked out using Table [1. Any rank-2 symmetric tensor, which arises in consideration of longitudinal flow, can be expressed as a linear combination of $L, V$ and $\Sigma$ (or alternatively, $L, V$ and $A$ ). Any similar rank-2 antisymmetric tensor can only be a scalar multiple of $\mathcal{A}$.

The vorticity tensor is defined as

$$
\omega^{\mu \nu}=\Delta^{\mu \alpha} \Delta^{\nu \beta} \frac{1}{2}\left(d_{\beta} u_{\alpha}-d_{\alpha} u_{\beta}\right),
$$

and is spacelike (orthogonal to $u$ ) and antisymmetric by construction. Due to its antisymmetry, $\omega^{\mu \nu}$ has to be proportional to $\mathcal{A}$. However, $\mathcal{A}$ is not orthogonal to $u$, so the only possible constant of proportionality is zero. In other words, vorticity vanishes for longitudinal flow. Another way to understand this is to note that $\omega^{\mu \nu}$, being an antisymmetric spacelike tensor, is equivalent (technically, dual) to an axial vector which is spacelike. Such a vector can be constructed by the three-dimensional vector product of two vectors. For longitudinal flow, there is only one spacelike vector $v$ which is intrinsic to the problem. Hence one cannot construct an axial vector. A tedious proof of the vanishing of the vorticity can also be given by direct manipulation of the definition.

In the presence of viscous shear but vanishing bulk viscosity, one of the hydrodynamic variables is the dissipative part of stress tensor, $\pi^{\mu \nu}$. The fact that it is symmetric can be derived from the symmetry of the stress tensor. Since it expresses shear, it is orthogonal to $u$. It is traceless since we have assumed bulk viscosity to vanish. Hence one can write uniquely,

$$
\pi^{\mu \nu}=\pi_{V}\left[V^{\mu \nu}-\frac{1}{2} \Sigma^{\mu \nu}\right]
$$

i.e., for longitudinal flow, the shear tensor is equivalent to a single scalar. Note that the tensor multiplying $\pi_{V}$ has the following properties-

$$
V^{\mu \nu}-\frac{1}{2} \Sigma^{\mu \nu}=\frac{3}{2} V^{\mu \nu}-\frac{1}{2} \Delta^{\mu \nu} \quad \text { and } \quad\left[V^{\mu \nu}-\frac{1}{2} \Sigma^{\mu \nu}\right]\left[V_{\mu \nu}-\frac{1}{2} \Sigma_{\mu \nu}\right]=\frac{3}{2} .
$$

We will need to consider derivatives of the basis tensors. This is facilitated by considering first the derivatives of the unit vectors. The condition $u^{\mu} u_{\mu}=1$ yields $u^{\mu} D u_{\mu}=0$, and therefore the orthogonality of $u$ and $v$. Now $D^{2} u^{\mu}$ can be written as a linear combination of $u$ and $v$. It is easy to work out that $D^{2} u^{\mu}=S^{2} u^{\mu}+(D S) v^{\mu}$. Furthermore, one can show that $D v^{\mu}=S u^{\mu}$. Two other special cases of interest for longitudinal flow are $d_{\mu} v^{\mu}=S$ and $v^{\mu} \widetilde{D} u_{\mu}=-\Theta$. For later use we also set down the actions of $D=u^{\mu} d_{\mu}$ and $\widetilde{D}=v^{\mu} d_{\mu}$ on any scalar field $f-$

$$
D f=f_{\tau} \cosh y+\frac{1}{\tau} f_{\eta} \sinh y \quad \text { and } \quad \widetilde{D} f=f_{\tau} \sinh y+\frac{1}{\tau} f_{\eta} \cosh y .
$$

Using the orthogonality of $u$ and $v$, we find the derivative

$$
\nabla_{\mu} u^{\mu}=d_{\mu} u^{\mu}=\Theta, \quad \text { where } \quad \nabla_{\mu} \equiv \Delta_{\mu}^{\nu} d_{\nu}
$$


Another derivative that appears repeatedly in the hydrodynamic equations is-

$$
\left\langle\nabla_{\mu} u_{\nu}\right\rangle \equiv \Delta_{\mu}^{\lambda} d_{\lambda} u_{\nu}+\Delta_{\nu}^{\lambda} d_{\lambda} u_{\mu}-\frac{2}{3} \Theta \Delta_{\mu \nu}
$$

where the notation $\langle\cdots\rangle$ denotes the traceless, symmetric part. Since this tensor is also orthogonal to $u$, one should be able to write (see eq. A2

$$
\left\langle\nabla_{\mu} u_{\nu}\right\rangle=\mathcal{D}\left[V_{\mu \nu}-\frac{1}{2} \Sigma_{\mu \nu}\right]
$$

where $\mathcal{D}$ is to be determined. Multiplying through by $V_{\mu \nu}$, one immediately finds

$$
\left\langle\nabla_{\mu} u_{\nu}\right\rangle=-2\left(v^{\lambda} \widetilde{D} u_{\lambda}+\frac{\Theta}{3}\right)\left[V_{\mu \nu}-\frac{1}{2} \Sigma_{\mu \nu}\right]=\frac{4}{3} \Theta\left[V_{\mu \nu}-\frac{1}{2} \Sigma_{\mu \nu}\right] .
$$

Now we are ready to write the derivatives of the basis tensors.

Since covariant derivatives of the metric tensor vanish, one has

$$
d_{\lambda} \Delta_{\mu \nu}=-\left(d_{\lambda} u_{\mu}\right) u_{\nu}-\left(d_{\lambda} u_{\nu}\right) u_{\mu}
$$

We examine its projections parallel and orthogonal to $u$. One of the parallel projections is

$$
D \Delta_{\mu \nu}=-S A_{\mu \nu}
$$

One of the orthogonal projections is

$$
\Delta_{\sigma}^{\mu} \nabla_{\rho} \Delta^{\sigma \rho}=\Delta_{\sigma}^{\mu} \Delta_{\rho}^{\lambda} d_{\lambda} \Delta^{\sigma \rho}=0
$$

For $V_{\mu \nu}$ one has

$$
d_{\lambda} V_{\mu \nu}=-\left(d_{\lambda} v_{\mu}\right) v_{\nu}-\left(d_{\lambda} v_{\nu}\right) v_{\mu}
$$

A parallel projection which we will use later is

$$
D V_{\mu \nu}=-\left(D v_{\mu}\right) v_{\nu}-\left(D v_{\nu}\right) v_{\mu}=-S A_{\mu \nu}
$$

One of the orthogonal projections that we need is

$$
\Delta_{\sigma}^{\mu} \nabla_{\rho} V^{\sigma \rho}=\left[S-d_{\rho} v^{\rho}\right] v^{\mu}
$$

Since $v_{\mu} v^{\mu}=-1$, one has $v^{\mu} \widetilde{D} v_{\mu}=0$, i.e., $\widetilde{D} v_{\mu}$ is parallel to $u$. As a result, one finds $\widetilde{D} v_{\mu}=\left(u^{\lambda} \widetilde{D} v_{\lambda}\right) u_{\mu}$, from which the last form of the derivative follows.

\section{APPENDIX B: THE EQUATIONS OF LONGITUDINAL HYDRODYNAMICS}

The dynamical equations are supplemented by the equation of state, which provides a relation between $\epsilon$ and $p$, and hence determines the entropy density $s=(\epsilon+p) / T$. Since the the hydrodynamic equations are valid only for a fluid in local thermodynamic equilibrium, or so close to it that linear response theory works, one may use the equation of state to eliminate one of $\epsilon$ and $p$ from hydrodynamics. A toy equation of state that we shall use is

$$
p(T)=c_{s}^{2} \epsilon(T)
$$

i.e., the speed of sound is independent of the temperature. The only value of $c_{s}^{2}$ which is strictly temperature independent is $c_{s}^{2}=1 / 3$. This is the appropriate value to use when the bulk viscosity has been neglected, since both are consequences of conformal symmetry. Since we use temperature independent $c_{s}^{2}$ in this paper, we use the above value whenever numerical work is performed. 


\section{The equation for energy}

The equation for energy in [14] is

$$
D \epsilon=-(\epsilon+p) \nabla_{\mu} u^{\mu}+\frac{1}{2} \pi^{\mu \nu}\left\langle\nabla_{\mu} u_{\nu}\right\rangle
$$

The identity in eq. (A3), used along with eq. (A8) gives

$$
\pi^{\mu \nu}\left\langle\nabla_{\mu} u_{\nu}\right\rangle=2 \pi_{V} \Theta
$$

Then, using the relation in eq. (A5), and the equation of state, one can write

$$
D \epsilon=-\left[\left(1+c_{s}^{2}\right) \epsilon-\pi_{V}\right] \Theta .
$$

\section{The momentum-balance equation}

The general form of the momentum-balance equation given in [14] is

$$
(\epsilon+p) D u^{\mu}=\nabla^{\mu} p-\Delta_{\sigma}^{\mu} \nabla_{\rho} \pi^{\sigma \rho}+\pi^{\mu \sigma} D u_{\sigma} .
$$

Note that each term is orthogonal to $u$. Using the definition of $v$ and the decomposition of the shear tensor in eq. (A2), we find that

$$
\pi^{\mu \sigma} D u_{\sigma}=S \pi^{\mu \sigma} v_{\sigma}=S \pi_{V} v^{\mu}
$$

Using the derivatives of the basis tensors in eqs. A11, A14, we can write

$$
\Delta_{\sigma}^{\mu} \nabla_{\rho} \pi^{\sigma \rho}=\Delta_{\sigma}^{\mu} \Delta_{\rho}^{\lambda}\left(d_{\lambda} \pi_{V}\right)\left[V^{\sigma \rho}-\frac{1}{2} \Sigma^{\sigma \rho}\right]-\frac{3}{2} \pi_{V} v^{\mu}\left[d_{\lambda} v^{\lambda}-S\right]
$$

The last term drops out because $d_{\lambda} v^{\lambda}=S$. Putting all this together, we can reduce the tensor equation to

$$
S(\epsilon+p) v^{\mu}=\nabla^{\mu} p-\left(d_{\lambda} \pi_{V}\right)\left[V^{\lambda \mu}-\frac{1}{2} \Sigma^{\lambda \mu}\right]+S \pi_{V} v^{\mu}
$$

Contracting with any spacelike tensor apart from $v$ would yield only terms in the directional derivative along that vector. But for longitudinal flow each such derivative is separately zero. Thus, the only nontrivial equation is obtained by contracting the above equation with $v$. This gives the entropy equation for longitudinal flow,

$$
S\left(\epsilon+p-\pi_{V}\right)+\widetilde{D}\left(p-\pi_{V}\right)=0
$$

\section{The equation for the shear tensor}

In [14] the equation for the shear tensor is given as

$$
\tau_{\pi} \Delta_{\alpha}^{\mu} \Delta_{\beta}^{\nu} D \pi^{\alpha \beta}+\pi^{\mu \nu}=\eta_{V}\left\langle\nabla^{\mu} u^{\nu}\right\rangle-2 \tau_{\pi} \pi^{\alpha(\mu} \omega_{\alpha}^{\nu)},
$$

where $\tau_{\pi}$ is a relaxation time, $\eta_{V}$ is the coefficient of shear viscosity, $\nabla^{\mu}=\Delta^{\mu \nu} d_{\nu}$, and $\omega^{\alpha \beta}$ is the vorticity tensor. Since the vorticity vanishes for longitudinal flow, we drop the last term.

Using eq. (A8) the term in the viscous coefficient becomes

$$
\eta_{V}\left\langle\nabla^{\mu} u^{\nu}\right\rangle=\frac{4}{3} \eta_{V} \Theta\left[V^{\mu \nu}-\frac{1}{2} \Sigma^{\mu \nu}\right] .
$$

For the first term, one can write

$$
\Delta_{\alpha}^{\mu} \Delta_{\beta}^{\nu} D \pi^{\alpha \beta}=\left(D \pi_{V}\right)\left[V^{\mu \nu}-\frac{1}{2} \Sigma^{\mu \nu}\right]
$$

since the projectors are orthogonal to the derivatives of the basis tensors in eqs. (A10, A13). The equation for shear then reduces to the scalar equation

$$
\tau_{\pi}\left(D \pi_{V}\right)+\pi_{V}=\frac{4}{3} \eta_{V} \Theta
$$




\section{APPENDIX C: EVOLUTION OF THE POWER SPECTRUM}

For the equations of sound we assume that a Fourier transformation decouples the individual Fourier modes. For each mode, the ODEs to be solved can be written in the form

$$
\frac{d \mathbf{x}}{d \theta}=M \mathbf{x},
$$

where $\mathbf{x}$ is a vector and $M$ a matrix. For ideal fluids, eqs. (15) can be written as a system of two coupled autonomous equations, so that $M$ is a $2 \times 2$ matrix. In the other cases (eqs. 30, 45 and its reduction in the ELNS limit) the equations are not autonomous, i.e., the time variable appears explicitly in $M$. However, the solution of eq. (C1) is straightforward and involves a matrix exponential. The asymptotic behaviour of the solution is controlled by the eigenvalue with the largest real part. We will assume that all the eigenvalues of $M$ have negative real part (at all times, in the non-autonomous case), so that at long times the solution decays. We are interested in whether transients grow.

Consider the real positive quantity $P(\theta)=\mathbf{x}^{\dagger} A \mathbf{x}$ where $A$ is a fixed matrix independent of $\theta$. Let $\mathcal{M}=M^{\dagger} A+A M$. Then

$$
\frac{d P}{d \theta}=\mathbf{x}^{\dagger} \mathcal{M} \mathbf{x}=\sum_{i}\left|x_{i}\right|^{2} \lambda_{i} \quad \text { where } \quad \mathbf{x}=\sum_{i} x_{i} \mathbf{v}_{i}
$$

in terms of the normalized eigenvectors, $\mathbf{v}_{i}$, and eigenvalues, $\lambda_{i}$, of $\mathcal{M}$. Since $P$ is real, $A$ is Hermitean. As a result, $\mathcal{M}$ is also Hermitean, and its eigenvalues are real. Transient growth in $P$ can take place for some initial conditions if and only if $\mathcal{M}$ has at least one positive eigenvalue. The largest growth in $P$ occurs when $\mathbf{x}$ is parallel to the eigenvector of $\mathcal{M}$ with largest (positive) eigenvalue.
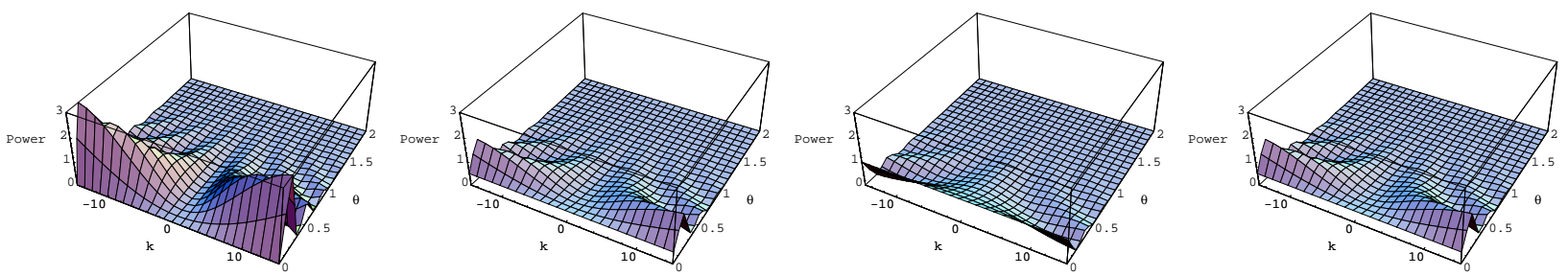

FIG. 16: (Color online) The power spectrum of fluctuations in energy density for the ideal fluid as a function of $k$ and $\theta$, for $\beta=0$ and $\alpha=0, \pi / 4, \pi / 2$ and $3 \pi / 4$ (from left to right). Note the lack of transient growth for $k=0$.
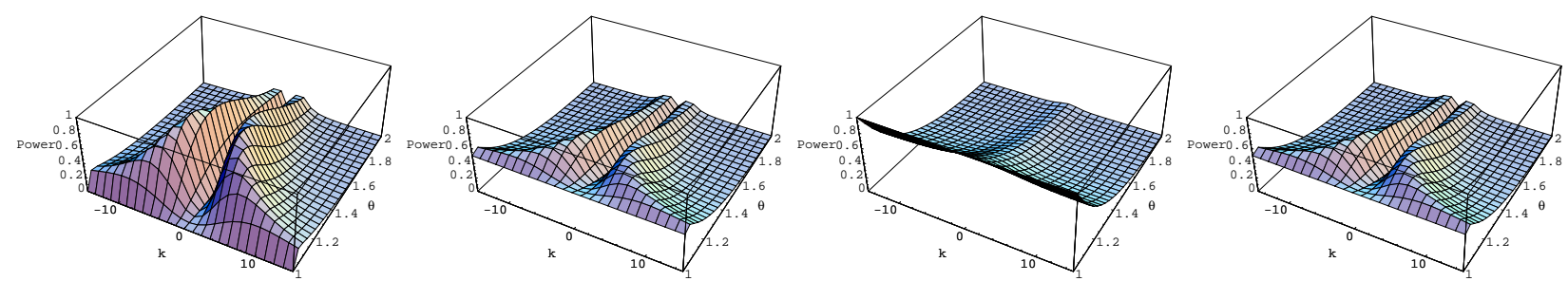

FIG. 17: (Color online) The power spectrum of fluctuations in energy density for the simple fluid in ELNS hydrodynamics as a function of $k$ and $\theta$, for $\beta=0$ and $\alpha=0, \pi / 4, \pi / 2$ and $3 \pi / 4$ (from left to right). Note the lack of transient growth for $k=0$.

When $A$ is chosen to be a multiple of the identity, then the eigenvalues of $\mathcal{M}$ are multiples of the real parts of the eigenvalues of $M$. Then, since the multiplying constant is positive, and we have assumed that the eigenvalues of $M$ have negative real parts, there can be no growth in $P$. In all other cases, transient growth of $P$ is possible.

In our application, we choose $A$ to be a projection operator on the first component of $\mathbf{x}$. As a result, $A M$ is just the top row of $M$ with other rows set to zero, and $\mathcal{M}$ is obtained by Hermitizing this. Now the eigenvalues of $\mathcal{M}$ have no simple relation with those of $M$. One can show that in general the rank of $\mathcal{M}$ is two, i.e., there are two non-vanishing eigenvalues. Unless all the off-diagonal terms in $\mathcal{M}$ are zero, one of the generically non-vanishing eigenvalues is positive and the other is negative. As a result, there is always transient growth in $P$. This is the reason for the peaks in Figures [5, 6] and 11. 
We complete the analysis of transient growth of $P$ for the ideal fluid (see eqs. 15). In this case we have

$$
\mathcal{M}=\left(\begin{array}{cc}
0 & -i k B \\
i k B & 0
\end{array}\right), \quad \text { with eigenvalues } \quad \lambda= \pm k B .
$$

Transient growth can take place. We can parametrize all initial conditions by an angle $\alpha$ and a phase $\beta$, by choosing $\mathbf{x}=(\sin \alpha \exp [i \beta], \cos \alpha)$. The evolution of the power spectrum of the energy obtained with specific initial conditions from numerical solutions of eqs. (15) are shown in Figure 16.

For the evolution of the power spectrum of $\chi^{1}$ in a simple fluid, one has

$$
\mathcal{M}=\left(\begin{array}{ccc}
-\frac{8}{3} & -4 i k & 1 \\
4 i k & 0 & 0 \\
1 & 0 & 0
\end{array}\right), \quad \text { with eigenvalues } \quad \lambda=0, \frac{1}{3}\left[-4 \pm \sqrt{25+144 k^{2}}\right]
$$

where we have taken $c_{s}^{2}=1 / 3$. Since one of the eigenvalues is positive, transient growth occurs. Note that the mode $k=0$ can also display transient growth. This analysis can be adapted to that for the Boltzmann fluid by replacing the constants in $\mathcal{M}$ by appropriate functions of $\theta$. However, the conclusions regarding transient growth carry over to that case.

For the power spectrum of $\chi^{1}$ in the ELNS approximation to the simple fluid, one finds that

$$
\mathcal{M}=-\left(\begin{array}{cc}
2 B & \frac{4}{3} i k \frac{1-c_{s}^{2}}{c_{s}^{2}} \\
-\frac{4}{3} i k \frac{1-c_{s}^{2}}{c_{s}^{2}} & 0
\end{array}\right), \quad \text { with eigenvalues } \quad \lambda=B \pm \frac{1}{3 c_{s}^{2}} \sqrt{9 c_{s}^{2} B^{2}+16 k^{2}\left(1-c_{s}^{2}\right)^{2}}
$$

Interestingly, the diffusive term, $c_{s}^{2} k^{2}$, drops out of the transient analysis for the energy density (it does appears in the transient analysis for $y$ ). For $k=0$ transients do not grow. This seems to be the main distinction between transient growth of energy density in ELNS and IS dynamics.

The numerical results in Figure 17 seem similar to the ideal fluid case at first sight. However, the diffusive character of the underlying equations is manifested in two ways. First, at fixed $k$, if one observes the time evolution, then one sees only a single instance of transient growth, unlike the quasiperiodic behaviour of $P_{\epsilon}$ in the ideal fluid. Second, at sufficiently large $k$ there is no transient growth, unlike the ideal fluid.

In the late time limit for a Boltzmann or conformal fluid one has, from eq. (47),

$$
\mathcal{M}=\left(\begin{array}{ccc}
2 p & -\frac{i k B u_{0}}{4} \mathrm{e}^{p \theta} & \frac{u_{0}}{4} \mathrm{e}^{p \theta} \\
\frac{i k B u_{0}}{4} \mathrm{e}^{p \theta} & 0 & 0 \\
\frac{u_{0}}{4} \mathrm{e}^{p \theta} & 0 & 0
\end{array}\right) \text {, with eigenvalues } \lambda=0, p \pm \frac{1}{4} \sqrt{16 p^{2}+\left(1+B^{2} k^{2}\right) u_{0}^{2} \mathrm{e}^{2 p \theta}} .
$$

Since one of the eigenvalues is positive, transient growth can take place.

[1] A. Hosoya, M. Sakagami and M. Takao, Ann. Phys., 154, 299, (1984);

A. Hosoya and K. Kajantie, Nucl. Phys., B, 250, 666, (1985);

G. Baym et al., Nucl. Phys., A, 525, 415C, (1991);

S. Jeon and L. G. Yaffe, Phys. Rev., D, 53, 5799, (1996);

P. Arnold, G. D. Moore and L. G. Yaffe, J. H. E. P., 11, 001, (2000);

G. Aarts and J. M. M. Resco, J. H. E. P., 4, 053, (2002);

P. Kovtun, D. T. Son and A. O. Starinets, J. H. E. P., 310, 064, (2003).

[2] C. Eckart, Phys. Rev., 58, 919, (1940).

[3] L. D. Landau and E. M. Lifshitz, Fluid Mechanics, Elsevier, New Delhi (2005).

[4] J. D. Bjorken, Phys. Rev., D, 27, 140, (1983).

[5] G. Baym et al., Nucl. Phys., A, 407, 541, (1983).

6] S. Kagiyama, A. Nakamura and A. Minaka, Prog. Theor. Phys., 76, 171 (1986)

[7] D. H. Rischke, S. Bernard and J. A. Maruhn, Nucl. Phys., A, 595, 346, (1995);

P. F. Kolb, J. Sollfrank and U. W. Heinz, Phys. Rev. C , 62, 054909, (2000);

D. Teaney, J. Lauret and E. V. Shuryak, Phys. Rev. Lett. , 86, 4783, (2001);

P. Huovinen et al., Phys. Lett., B, 503, 58, (2001);

T. Hirano and K. Tsuda, Phys. Rev. C , 66, 054905, (2002);

P. F. Kolb and R. Rapp, Phys. Rev. C , 67, 044903, (2003).

[8] See for example, the treatment in P. J. E. Peebles and Bharat Ratra, Rev. Mod. Phys. , 75, 559, (2003). 
[9] P. Danielewicz and M. Gyulassy, Phys. Rev., D, 31, 53, (1985);

D. Teaney, Phys. Rev. C , 68, 034913, (2003).

[10] M. Prakash, M. Prakash, R. Venugopalan and G. Welke, Phys. Rep., 227, 321, (1993).

[11] A. Muronga, Phys. Rev. Lett. , 88, 062302, (2002) and Phys. Rev. C , 69, 034903, (2004).

[12] A. Muronga and D. H. Rischke, nucl-th/0407114.

[13] U. Heinz, H. Song and A. K. Chaudhuri, Phys. Rev. C , 73, 034904, (2006).

[14] R. Baier, P. Romatschke and U. A. Wiedemann, Phys. Rev. C, 73, 064903 (2006), and Nucl. Phys., A, 782, 313 (2007).

[15] M. Lubinsky and E. Shuryak, arXiv:0704.1647

[16] P. Romatschke and U. Romatschke, arXiv:0706.1522.

[17] A. Dumitru, E. Molnar and Y. Nara, arXiv:0706.2203.

[18] H. D. Weymann, Am. J. Phys., 35, 488, (1967);

D. C. Kelly, Am. J. Phys., 36, 585, (1968);

N. G. van Kampen, Physica, 46, 315, (1970).

[19] W. Israel, Ann. Phys., 100, 310, (1976);

J. M. Stewart, Proc. Roy. Soc., A 357, 59, (1977);

W. Israel and J. M. Stewart, Ann. Phys., 118, 341, (1979);

W. A. Hiscock and L. Lindblom, Ann. Phys., 151, 466, (1983).

[20] T. Koide, G. S. Denicol, Ph. Mota and T. Kodama, hep-ph/0609117.

[21] M. Ichiyanagi, Prog. Theor. Phys., 84, 810, (1990);

T. Koide, Phys. Rev., E, 75, 060103(R) (2007).

[22] A. Muronga, Phys. Rev. C , 76, 014909, (2007).

[23] A. Muronga, Phys. Rev. C , 76, 014910, (2007).

[24] L. D. Landau and E. M. Lifshitz, Classical Theory of Fields, Elsevier, New Delhi (2005).

[25] H. Kouno, M. Maruyama, F. Takagi and K. Saito, Phys. Rev., D, 41, 2903, (1990).

[26] R. Baier and P. Romatschke, nucl-th/0610108.

[27] R. Jackiw in Current Algebra and Anomalies, eds. S. B. Treiman et al., p. 168, World Scientific, Singapore, 1985.

[28] S. Gupta, Pramana, 61, 877, (2003).

[29] B. Beinlich et al., Eur. Phys. J., C 6, 133, (1999);

Y. Namekawa et al., Phys. Rev., D, 64, 074507, (2001);

A. Ali Khan et al., Phys. Rev., D, 64, 074510, (2001).

[30] P. Arnold, C. Dogan and G. D. Moore, Phys. Rev., D, 74, 085021, (2006).

[31] R. V. Gavai, S. Gupta and S. Mukherjee, Phys. Rev., D, 71, 074013, (2005).

[32] D. Kharzeev and K. Tuchin, arXiv:0705.4280.

[33] M. P. Heller and R. A. Janik, hep-th/0703243.

[34] S. Gupta, Phys. Lett., B, 597, 57, (2004);

H. B. Meyer, eprint arXiv:0704.1801 [hep-lat].

[35] S. Gupta, hep-ph/0507210;

R. S. Bhalerao et al., Phys. Lett., B, 627, 49 (2005).

[36] I. S. Gradshteyn and I. M. Ryzhik, Tables of Integrals, Series, and Products, Academic Press, San Diego, 2000.

[37] http://mathworld.wolfram.com/MeijerG-function.html

[38] V. I. Arnold, Ordinary Differential Equations, The MIT Press, Cambridge, Massachusetts, 1973.

[39] See, for example, A. C. King, J. Billingham and S. R. Otto, Differential Equations, Linear, Nonlinear, Ordinary, Partial, Cambridge University Press, Cambridge UK, 2003. 